\title{
Experimentelle Untersuchungen über die Heilungsvorgänge von Lederhantwunden.
}

\author{
Von \\ Dr. Emil Krückmann, \\ Privatdocenten und Assistenten zu Leipzig. \\ (Aus der Universitäts-Augenklinik zu Leipzig.) \\ Mit Tafel III-V, Fig. 1-8.
}

Während unsere Kenntnisse über die Heilung von Hornhautwunden durch eine Anzahl trefflicher Arbeiten geklärt sind, kann ein Gleiches vom Heilungsvorgang der Lederhautverletzungen nicht gesagt werden.

Einige Untersucher liefern zwar eine Illustration des Heilungsverlaufes sämmtlicher Augenhäute, doch verfügen sie nur über eine kurze Beobachtungszeit, sowie über eine relativ geringe Anzahl von Experimenten. Andere beschränken sich ausschliesslich auf die Mittheilung der histologischen Veränderungen an der Netzhaut. Hierdurch erscheint es erklärlich, dass die Publicationen meistens nur Uebersichtsbilder liefern, sowie, dass die Angaben der meisten Untersucher vielfach miteinander differiren.

Fin kurzer Rückblick mag genïgen, das Wesentliche aus den Ergebnissen der verschiedenen Beobachter vorzufiuhren.

Penetrirende Wunden der Augenhänte werden nach Lubinsky ${ }^{1}$ ) durch Neuproduction von Aderhaut- und Bindehantgewebe geschlossen.

1) Alex. Lubinsky, Ueber die den Augapfel penetrirenden Wunden nach an Kaninchen ausgeführten Experimenten. Archiv $f$. Ophthalm. Bd. 13. Abth. 2, S. 377. 
Nach Schunkitz Miyshita ${ }^{1}$ genügt bei isolirter Verletzung der Lederhaut die Wucherung der sklerazellen allein, um eine völlige Heilung zu Stande zu bringen; dagegen soll sich bei Continuitätstrennungen der inneren Augenhäute mit Schonung der Lederhaut fast ausschliesslich die Aderhaut am Heilungsprocess betheiligen.

Roth ${ }^{2}$ ) nimmt eine gemeinschaftliche Thätigkeit der Lederhaut und des Glaskörpers an.

Nach Baquis ${ }^{3}$ ) kommt der Wrudversehluss dureh ein Zellmaterial zu Stande, dessen Abstammung in der Sklera und in dem peribulbären Gewebe zu suchen ist.

Tepljaschin ${ }^{4}$ ) glaubt die Hauptlieferung des Keimgewebes aus den Zellelementen der Chorioidea, sowie des peribulbären Gewebes ableiten zu müssen, doch hält er eine Mitbetheiligung der Sklera und besonders des Glaskörpers nicht für ausgeschlossen.

Schliesslich erschien vor kurzer Zeit noch ein Aufsatz von E. Franke ${ }^{5}$ ), welcher sich gleichfalls mit der Heilung perforirender Lederhautwanden beschäftigt. Nach ihm sind es „die zell- und gefässreichen Gewebe der Episklera und der Aderhant, welche im wesentlichen durch die Proliferation never Zellen sich an der Narbenbildung betheiligen. Die Sklera liefert nur Fibrillen, welche aus den fixen Bindehautgewebskörperchen hervorgegangen $\left.\sin d^{\prime \prime 6}\right)$.

1) Schunkitz Miyshita, Experimentelle Studien über die Verheilung von Lederhaut-. Aderhant- mod Netzhautwunden. Inaug.Dissert. Würzburg 1888.

2) Roth, Beiträge zur Kenntniss der varicösen Hypertrophie der Nervenfasern. Virchow's Archiv. Band 55. S. 212.

3) Baquis, Etude expérimentale sur les rétinites en rapport avee la réaction irritative des divers éléments rétineux. Ziegler's Beitr. Bd. 4. S. 265.

4) Tepljaschin, Zur Kenntniss der histologisehen Veränderungen der Netzhaut nach experimentellen Verwundungen. Arch. f. Augenklinik, Bd. 28. S. 354.

5) E. Franke, Ueber die histologischen Vorgänge bei der Heilung perforirender Lederhautwunden. Arch. f. Ophthalm. Bd. 41, Abth. 3. S. 30.

6) Während der Correctur erfuhr ich durch die Abhandlungen von Uhth off und Axenfeld in den Ergebnissen der speciellen pathologischen Morphologie und Physiologie von Lebarseh, S. 253, dass 
Exp. Untersuch. über die Heilungsvorgänge von Lederhautwunden. 295

Da dieser Autor nur mit Kaninchen experimentirte, die Wunden nicht länger als 18 Tage beobachtete und seine Untersuchung ausschliesslich auf die beiden erwähnten, der Lederhaut benachbarten Schichten concentrirte, so ist auch hier, trotz der ausführlichen und genauen Schilderung der Befunde, nur der Theil eines Ganzen dem Studium unterzogen worden.

Die Entstehung meiner Arbeit verdanke ich der Anregung meines hochverehrten Chefs und Lehrers, des Herrn Geheimrath Sattler, dem ich auch an dieser Stelle für die vielfachen Opfer an Zeit und Mühe meinen herzlichsten Dank sage.

Da eine sorgfältige und gesichtete Schilderung der feineren Einzelheiten, welche bei der Wundheilung als ausschlaggebende Momente in Betracht kommen, im Interesse einer wissenschaftlichen Controle und eines gerechtfertigten Urtheils gefordert werden musste, so war es wünschenswerth, ein Gesammtbild der Heilungsvorgänge zu erhalten, um für die gleichen Verhältnisse am menschlichen Auge Parallelen und Analogieen constituiren zu können.

Es wurde daher nothwendig, an mehreren Thierarten unter den verschiedensten Bedingungen den Heilungsverlauf zu studiren. Die erstere Anforderung erfuillte ich durch die Benutzung von mehr als hundert Versuchsthieren der fünf Species: Kaninchen, Meerschweinchen, Hunden, Katzen und Ratten. Dem zweiten Postulat glaube ich dadurch genügt zu haben, dass ausser der wechselnden Schnittrichtung, durch die Herbeiführung von Prolapsen, Anlegung von Nähten, Bildung von Gewebslappen, Lospräpariren von Bulbushüllen etc. die mannigfaltigsten Eventualitäten für den Heilungsvorgang geschaffen wurden.

durch experimentelle Untersuchungen von $\mathrm{Krebs}$ und Herrnheiser gleichfalls der Wundverschulss an den Augenhäuten studirt worden ist. Ich werde Gelegenheit nehmen diese Literatur in einer späteren Arbeit genau zu beriicksichtigen. 
Die Sklera wurde mit einem Graefe'schen Messer durchtrennt. Die Wundlänge betrug bei den Kaninchen, Hunden und Katzen $5-6 \mathrm{~mm}$, bei den Meerschweinchen 3-4 mm und bei den Ratten 2-3 mm. Sehr viele Augen wurden frisch untersucht; doch wurde die grössere Anzahl fixirt und gehärtet. Zur Fixation kamen die gebräuchlichsten Flüssigkeiten in Anwendung: Alkohol absol., Müllersche und Flemming'sche Lösung, Sublimat, Pikrinsäure, Formalin. Eingebettet wurde in Paraffin und Celloidin. Als Färbemittel dienten Osmiumsäure, Hämatoxylin, Eosin, Jodhämatoxylin, Safranin, Alaun- und Boraxcarmin, die Weigert'sche Bakterien- und Fibrinfärbung, die Löfflersche Methylenblaulösung, sowie das Färbegemisch von Bergoncini. Untersucht wurden die Präparate in Canadahalsam und Glycerin.

Die Beobachtungszeit der Heilungsdauer erstreckte sich von 4 Stunden bis auf 120 Tage.

Die Wundheilungen verliefen aseptisch.

Grob anatomischer und klinischer Befund.

Unmittelbar nach der Verletzung entsteht in der Regel eine Pupillenverengerung, welche nach 2-4 Stunden vorübergeht. Der Augendruck ist stets herabgesetzt, wovon ich mich durch tonometrische Messungen deutlich überzeugen konnte. Die vordere Kammer ist meistens vertieft. Zuweilen ist geringes Irisschlottern erkennbar. Im Glaskörperaume sieht man eine ausgedehnte von der Wunde stammende Blutung, welche ein genaues Ophthalmoskopiren unmöglich machte. Liegen die Wunden im vorderen Augenabschnitt, so bemerkt man deutlich, wie die Bindehaut, das episklerale Gewebe, sowie die Interstitien der quergestreiften Muskulatur in weiterer Umgebung der Verletzung hyperämisch werden. Zugleich tritt Chemosis auf. Diese letzteren beiden Erscheinungen erreichen ihren Höhepunkt nach 
Exp. Untersuch. über die Heilungsvorgänge von Lederhautwunden. 297

6-12 Stunden und halten ca. $24-36$ Stunden an. Nur sehr selten wird die Hornhaut matt; doch erhält sie ihren Glanz schon am zweiten Tage wieder.

\section{Mikroskopischer Befund.}

Vier bis sechs Stunden nach der Durchschneidung der Augenhäute findet man bei jeder Thierart folgendes Bild:

Alle Augenhäute stehen am Wundrande von einander $a b$, am ausgedehntesten die Netzhaut. Der Glaskörper ist vorgefallen. In der Nähe der $W$ unde besteht ausgesprochene Hyperämie. Die Wundränder sind verbreitert. Die Ursache der letzteren Erscheinung beruht in einer serösen Durchtränkung, sowie in der aufgehobenen Spannung der Gewebe verbunden mit einer mehr oder weniger ausgebreiteten Blutung. Die präexistirenden Kerne sind an den Wundrändern durch die Färbung nicht kenntlich zu machen. Der ganze Wundcanal ist von einer blassen, ca. 0,2-1,0 mm breiten Zone eingerahmt, welche die Zeichen der Coagulationsnekrose trägt (Fig. 1). Die einzelnen Augenhäute zeigen diese nekrotischen Veränderungen fast in gleicher Ausdehnung; nur variiren die letzteren in ihrer Form, ent sprechend dem specifischen Aufbau der Gewebe. Am auffallendsten sind sie in der Sklera, wo die Fibrillenenden ein franzenartiges Aussehen haben, welches einem aufgedrehten Bindfaden nicht unähnlich erscheint.

Einige Stunden später bemerkt man an den Nachbarzellen dieser kernarmen Zone gleichfalls Veränderungen. Die Endothelien der angrenzenden Suprachorioidea (Fig. 20.) und der Tenon'schen Kapsel sind geschwollen und gequollen. Sehr viele haben ein scholliges Aussehen erlangt und sind desquamirt. Thre Kerne sind entweder gross und blass, oder geschrumpft und feinkörnig zerfallen. Die Sklerazellen zeigen im Bereiche der erwähnten Zone die gleichen Veränderungen wie die Endothelien; zum Theil liegen ihre Kerntrümmer frei in den Saftbahnen. Frisch unter- 
sucht und mit specifischen Agentien behandelt zeigen sich die meisten in fettiger Degeneration begriffen.

Aehnlich verhält sich bei entsprechender Schnittlage die glatte Muskulatur des Ciliarkörpers.

Am intensivsten verändert zeigt sich die Retina. Ausser den zuerst von Berlin ${ }^{1}$ ) richtig erkannten und geschilderten raricösen und kugelförmigen Degenerationsformen der Sehnervenfasern (Fig. 3 d) ist fast in allen Nervenzellen eine feinkörnige, staubartige oder vacuoläre Metamorphose erkennbar. Bei Weitem am ausgesprochensten zeigt sich dieselbe in der Ganglienzellenschicht (Fig. $2 \mathrm{n}, 3 \mathrm{~g}$ ). Nicht auffindbar war sie an den Zapfen und Stäbchen.

Die Pigmentepithelzellen sind am Schnittrande von ungleicher Grösse und Form. Fast alle sind von der Aderhaut abgehoben. Meistens sind sie gequollen und entweder feinkörnig getruibt oder mit Fetttröpfchen durchsetzt (Fig. $2 \mathrm{~g}, 3 \mathrm{e})$.

Die Kerne sind blass und gleichfalls gequollen. Einige zeigen nach der Desquamation ihre Conturen nur wenig verändert. Das Färbevermögen ist verringert, resp. ganz erloschen. Zuweilen ist die friuhere Zelle nur noch in schattenhafter und unregelmässiger Begrenzung als Scholle auffindbar. Die kleinen Pigmentsplitterchen liegen im Protoplasma und erinnern in ihrem Verhalten an die von C. Hess ${ }^{2}$ ) bei Naphthalininjectionen erhaltenen Bilder. Meist sind sie nur spärlich vorhanden, oft haben sie die Zelle vollständig verlassen und liegen dann frei im subretinalen Raum (Fig. 2 i). In einiger Entfernung von der Wunde,

1) Berlin, Ueber den Gang der in den Glaskörperraum eingedrungenen fremden Körper. Archiv f. Ophthalm. Bd. 13. Abth. 2, S. 295 .

2) C. Hess, Ueber die Naphthalinveränderungen im Kaninchenauge und über Massagecataract. Bericht der 19. Versammlung der Ophthalm. Gesellschaft zu Heidelberg, 1887. \$. 54. 
Exp. Untersuch. über die Heilungsvorgänge von Lederhautwunden. 299

ca. 1-3 mm, nehmen auch diese Veränderungen $a b$, und besonders auf dem Flachschnitt zeigt sich wieder das gleichmässige, ungestörte Mosaik der Pigmentepithelien. Auch glaube ich mich nicht in der Annahme zu täuschen, dass die Müller'schen Stützfasern sich gleichfalls fettig degenerativ verhielten. Ueber die Neurogliazellen kann ich nichts Bestimmtes aussagen.

Diese degenerativen Veränderungen konnten nur in sehr begrenzter Zeit an ungefärbten frischen Präparaten constatirt werden, denn eine klare Uebersicht, sowie ein scharfes Bild mit exacter Differenzirung der einzelnen Zellarten wurde bald verwischt durch die auf der Bildfläche erscheinenden Leukocyten, welche gleichfalls massenhafte Fetttröpfchen enthielten. Mit dem Auftreten dieser Zellen hat sich die Hyperämie zu einer prallen Fiillung und starken Ausdehnung der kleinen Gefässe und Capillaren entwickelt, so dass dieselben gleichsam natürlich injicirt erscheinen. Desgleichen hat die seröse Durchtränkung der Gewebe bedeutend zugenommen (Fig. 1). Auf und neben den Wundrändern werden Fibrinbildungen sichtbar.

Diese Erscheinungen werden aber fast ganz in den Hintergrund gedrängt durch die Ueberschwemmung von Leukocyten, welche das der Wunde benachbarte Territorium fast ganz allein occupirt zu haben scheinen. Die einzelnen Leukocytengruppen sind gegen den Wundrand gerichtet. (Fig. 1.) Man erkennt deutlich, wie sie nach der typischen Randstellung (Fig. 1 a) sieh durch die Gefässe hindurchzwängen, und von allen Seiten zum Wundcanal begeben; indem sie sich entweder in die meist erweiterten Saftcanälchen der Sklera einklemmen, (Fig. 1d) und durch dieselben gegen die Wundfläche vorschwärmen, oder von aussen resp. innen die Wundränder in zarter Linie sowie dichten Trupps garmiren. (Fig. 1c.) Die Maschen der Suprachorioidea sind mitunter ganz von Leukocyten ausgefiillt. 
Bei entsprechender Schnittrichtung wird dass grösste Contingent von der Aderhaut und dem vorderen episkleralen Gewebe geliefert. Schon weniger betheiligen sich - bei Wunden in dieser Gegend - der Ciliarkörper, sowie die Interstitien der quergestreiften Muskulatur; und es frappirt geradezu die Indolenz der Lederhaut, welche sich vollkommen inactiv zu verhalten scheint. Dieser Befund verliert allerdings sein Befremdendes, wenn man erwägt, dass die Sklera wegen ihrer Gefässarmuth zum Passivsein gezwungen ist, sowie dass das Caliber und die Wandung der Rami perforantes einen Austritt von Leukocyten erschweren. Nur bei den Katzen und Hund finden sich übereinstimmende Veränderungen. Hier betheiligen sich die spärlichen Skleragefässe direct, und es ist auffällig, wie gerade bei diesen beiden Thierarten die Skleragefässe von den Leukocyten in grosser Anzahl verlassen werden. Die Lederhaut ist hier ganz besonders dick und in ihr eine relativ grosse Anzahl von Gefässen vorhanden.

Ausserordentlich interessant und im höchsten Grade lehrreich ist der sich überall wiederholende Befund, dass die Leukocyten dann am ausgiebigsten und intensivsten in und neben den Wundrändern angehäuft werden, wenn die Schnittflächen des episkleralen Gewebes und der Aderhaut nicht retrahirt sind, und mit dem skleralen Wundcanal in einer Ebene liegen. In einem Falle, wo ein Theil der Chorioidea prolabirt war, und sich über die Conjunctiva geschlagen hatte, war die Infiltration von Leukocyten so diffus, dass die Grenzen der einzelnen Augenhäute zum Theil verwischt waren (Fig. 1 links). Erhalten sich die Wundränder des episkleralen Gewebes, der Lederhaut und der Aderhaut in gleichem Niveau, so ist im vorderen Augenabschnitt der intermediäre Theil des skleralen Wundcanals, ceteris paribus, der zuletzt von den Leukocyten besuchte. Hiermit steht im Finklang, dass die Leukocyteninfiltration der Lederhaut nach Abtragung der angrenzenden Episkleral- 
Exp.Ưntersuch. über die Heilungsvorgänge von Lederhautwunden. 301

schichten, sowie nach Entfernung und Loslösung der benachbarten Aderhaut langsam und in ungleichmässiger Weise erfolgt (Fig. 1 rechts); sowie dass in der ersten Zeit als einzige Veränderung nur eine Verbreiterung der nekrotischen Zone aufzufinden ist. Bei Schnitten in der hinteren Bulbushälfte wird die Lieferung des Leukocytenmaterials in erster Linie von der Aderhaut übernommen; denn das Gewebe der Tenon'schen Kapsel ist hinten blutarm, und die Retinalgefässe stehen wegen ihrer geringen Mitbetheiligung praktisch ausser Concurrenz. Vorne und besonders in der Nähe der äusseren Muskelansätze tritt die Leukocytenemigration aus den extraskleral gelegenen Theilen ausserordentlich deutlich hervor. Dagegen ist sie in den hinteren Lederhautabschnitten auf ein Minimum reducirt, und erlangt nur dann eine grössere Ausdehnung, wenn sich die Wunde nahe der Insertion des Musc. retractor bulbi befindet. Alle diese Erscheinungen werden verständlich, wenn man bedenkt, dass die Leukocyteninfiltration vom Blutreichthum der Gewebe abhängig ist, wobei ergänzend hinzugefügt werden muss, dass die durch die Operation bedingten Lageverschiebungen der blutreichen Augengewebe bestimmend auf die Leukocytenwanderung einwirken können, so dass das eine Gewebe für das andere einzutreten im Stande ist (Fig. 1 links).

Ist Glaskörper prolabirt, so wird der äussere Rand der Glaskörperhernie gleichsam kranzförmig von den weissen Blutzellen umsäumt; doch dringen dieselben nur spärlich und allmählich in das vorgefallene Glaskörpergewebe ein. An der äusseren Wundpforte etablirt sich meistens ein dichtes Leukocytendepot, ron dem aus die einzelnen Zellen in der beschriebenen Weise am Wundrand entlang wandern, um sich mit ihren von innen kommenden Partnern im Wundeanal und in den skleralen Saftlücken zu vereinigen.

Die Leukocyten sind fast ausnahmslos polynucleär; sie 
zeigen nierenförmige und gelappte Kerne. Frisch untersucht enthalten dieselben reiche Fetttröpfchen, sowie mitunter Splitterchen und Körnchen von Pigment.

\section{Epikrise.}

Bis zum Ende des ersten Tages wird das histologische Bild fast allein durch entzündliche Vorgänge construirt. Die Exsudation mit der Auflockerung der Gewebe, die Leukocyteninfiltration und die Fibrinbildung beherrschen die Situation und damit das mikroskopische Gesichtsfeld (Fig. 1). Die Begleiterscheinungen der Verletzungen, wie Blutungen und Vorfälle, sowie die S. 297 ff. geschilderten Degenerationszustände der präformirten Gewebselemente, stehen diesen Processen gegenüber zurïck. Die Entzündungserscheinungen und die der Coagulationsnekrose analogen Veränderungen, welche in den ersten Stunden nach der Verletzung auftreten, zeigen eine solche Uebereinstimmung mit den Befunden, welche nach Continuitätstrennungen an anderen Organen besonders von Ziegler und seinen Schülern beschrieben worden sind, dass eine weitere eingehende Besprechung ihrer einzelnen Vorgänge überflüssig ist. Es geniige die Mittheilung, dass allem Anschein nach die Stärke der Exsudation in ein directes, und die Ausdehnung der Nelzrose in ein umgekehrtes Verhältniss zu dem Blutreichthum der skleralen Nachbargewebe gesetzt werden kann.

Diese entzündlichen Erscheinungen nehmen im Laufe des zweiten Tages ab und statt ihrer treten die degenerativen Veränderungen wieder in den Vordergrund.

\section{Zweiter Tag.}

Mikroskopischer Befund.

Man erkennt jetzt überall in der Wundnähe blasse unregelmässige Protoplasmareste, mehr oder weniger intensiv gefärbte Kerntrümmer, sowie Splitter und Körnchen 
Exp. Untersuch. über die Heilungsworgänge von Lederhautwunden. 303

von Pigment (Fig. $2 \mathrm{~h}, \mathrm{i}, \mathrm{k}$ ). Am meisten verändert ist wiederum die Retina.

Dieselbe zeigt am Wundcanal eine Zunahme ihrer Abhebung (Fig. 3), mitunter ist sie in Falten gelegt. Die varicösen Verdickungen der Nervenfasern haben zugenommen. In der Ganglienzellschicht sind die Zellen geschwollen und glasig aufgetrieben (Fig. $3 \mathrm{~g}, 5 \mathrm{c}$ ). Das Protoplasma zeigt häufig Vacuolen. Der Kern ist blass, kaum färbbar. Andere Zellen sind verkleinert, geschrumpft, bröckelig zerfallen (Fig. 2 n). Einige sind völlig verschwunden, so dass zwischen den Stützfasern leere Plätze entstehen (Fig. $3 \mathrm{f}, 5 \mathrm{~b}, 1$ ), Die Körnerschichten sind verschmälert (Fig. 2, 3, 5). Ihre feineren histologischen Degenerationsformen lassen sich nur schwer erkennen. Winzige Chromatinkügelchen und kleinste kaum färbbare Körnchen sind oft die einzigen Ueberreste. Dio Stäbchen und Zapfen sind in feinkörnige Massen verwandelt (Fig. $2 \mathrm{k}$ ). Mitunter hat man den Eindruck, als wenn am Aussenglied der Stäbchen eine Verlängerung auftritt. Die desquamirten Pigmentepithelzellen zeigen die S. 298 geschilderten destructiven Veränderungen. Dieselben sind jetzt sowohl im Wundcanal, als auch rareficirten Netzhautgewebe auffindbar.

An der Aderhaut sind degenerative Veränderungen am ausgesprochendsten in der Suprachorioidea sichtbar. Die Stromapigmentzellen sind pigmentarm und geschrumpft; zum Theil sind sie in krümelige Massen eingebettet. Thre Fortsätze sind eingezogen oder abgestossen. Die Endothelien der Suprachorioidea sind in grosser Ausdehnung in der S. 297 beschriebenen Weise verändert (Fig. 20). Erst in einiger Entfernung, 1-2 $\mathrm{mm}$, vom Wundcanal zeigen sie wieder ihre normale Configuration. Die Zellen des Tapetum lucidum gehen gleichfalls in grosser Ausdehnung zu Grunde. Dieselben verlieren ihren glänzenden Inhalt, quellen auf, werden durchsichtig und kugelig. Der Kern wird blass, 
legt sich excentrisch und quillt gleichfalls sehr häufig (Fig 4c). Schliesslich zerfällt die ganze Zelle ohne besondere Spuren zurückzulassen.

Die Epithelien der Conjunctiva sind in Degeneration, sowie in Desquamation begriffen, und ähnliches findet sich bei geeigneter Schnittführung in den Epithelzellen der pars ciliaris retinae.

Selbstverständlich verstehen sich alle diese Veränderungen nur auf die Umgebung der Wunde.

Im Laufe des zweiten Tages nehmen die Leukocyten ab. Das entzündliche Exsudat ist kaum mehr sichtbar. Die Fibrinbildungen sind zum grössten Theile verschwunden. Auch die Blutungen sind kleiner geworden; mitunter erinnern die rothen Blutkörperchen nur noch als Schatten an eine frühere Haemorrhagie. Die Wundränder sind geglättet (Fig, 2). Der Detritus und die fibrinösen Entzündungsproducte sind durch die Leukocyten eliminirt worden. Die Wundflächen sind histologisch durch Resorption der nekrotischen Gewebsenden für den Heilungsprocess vorbereitet, ähnlich wie einem unregelmässig gerissenen Drahte durch Feile und Sandpapier vor dem Zusammenlöthen eine glatte Fläche gegeben wird. Die Thätigkeit der weissen Blutzellen ist jetzt zum grössten Theile erschöpft; sie selbst sind überflüssig und unsichtbar geworden.

\section{Epikrise.}

Die Mobilmachung der Leukocyten zur Hinwegschaffung der abgestossenen Gewebsbestandtheile und zur Glättung der Wundränder ist ein stereotyper Process nach jeder Verletzung und die unbedingte Praemisse einer exacten Wundheilung. Im Verhältniss zu anderen Organen mag als besonders bemerkenswerth die grosse Intensität in den degenerativen Veränderungen der hochdifferenzirten Zellen notirt werden, wie sie hier durch den ausgedehnten Schwund 
Exp. Untersuch. über die Heilungsvorgänge von Lederhautwunden. 305

der nervösen Elemente der Pigmentepithelien und der Tapetumzellen zu Stande kommt.

Bei grober Skizzirung entspricht das histologische Bild des ersten Tages den exsudativen und das des zweiten den degenerativen Vorgängen, doch gehen beide Processe ineinander über. $\Delta$ uch die Degenerationsveränderungen schliessen mit dem zweiten Tage nicht ab, nụ treten sie zu dieser Zeit am deutlichsten hervor, zumal die Entzündungserscheinungen weniger störend wirken und die Bildung von jungen Granulationsstellen sich erst im Anfangsstadium befindet. Auch dieser Befund deckt sich in der Hauptsache mit den Ergebnissen der Ziegler'schen Schule bei Wunden an anderen Organen.

\section{Dritter Tag bis zum Fnde der ersten Woche.}

Grob anatomischer und klinischer Befund.

Am dritten Tage beschränkt sich die Hyperämie der extraskleral gelegenen Schichten auf die Umgebung der Wunde. Die Wundränder sind miteinander verklebt. Die Hornhaut ist klar. Die Pupille reagirt prompt. Der Tonus ist in der Regel wieder hergestellt. Im Augeninnern ist wegen der intraocularen Blutung nichts wesentliches erkennbar. Im Laufe der ersten Woche bleiben diese Verhältnisse fast unverändert. Der intraoculare Druck stellt sich völlig wieder her. Die Glaskörperblutungen werden kleiner, doch sind sie noch gross genug, um ein Ophthalmoskopiren $\mathrm{zu}$ verhindern.

\section{Mikroskopischer Befund.}

Wie soeben gesagt beginnt im Laufe des zweiten Tages die formative Thätigkeit der fixen Gewebszellen.

In der Netzhaut treten am Wundcanal in der Nervenfaserschicht kleine blasige Kerne mit reichlichem, intensiv gefärbtem Chromatin auf (Fig. 21,3a, 5 f). In den Müller- 
schen Stützfasern erkennt man Mitosen in allen Stadien (Fig. 5d). Die Zellen der Adventitien sind vermehrt und enthalten grosse blasige Kerne (Fig. $3 \mathrm{~b}, 5 \mathrm{~g}$ ).

In der Aderhaut wuchern besonders die Adventitiazellen der grossen Gefässe (Fig. 2 e, 3, 6, 8). Desgleichen sind im elastischen Faserwerk der Suprachorioidea - und besonders auf der Innenfläche der Sklera - neben Karyomitosen (Fig.2f) grosse ovale, blasige Kerne auffindbar, welche sich deutlich von den länglichen, flachen normalen Endothelkernen abheben.

Am schönsten sieht man den Beginn der Proliferation, sowie die verschiedensten Kerntheilungsfiguren in der Sklera (Fig. 2 c, d, q), dem episkleralen Gewebe, der Conjunctiva und den Interstitien der willkürlichen Muskulatur. Mitunter findet man in einem Gesichtsfelde alle Stadien der Karyokinese.

Relativ indolent verhält sich der verletzte Ciliarkörper. Allerdings findet man auch hier Proliferationsvorgänge, doch sind dieselben nur verschwindend gering. Bemerkenswerth ist schon jetzt ein Längerwerden der Cylinderepithelien auf der pars ciliaris retinae.

Im Allgemeinen steht die numerische Zellproduction im directen Verhältniss zum Dickendurchmesser der einzelnen Augengewebe, sowie zur Grösse der Verletzung. Andererseits macht es den Eindruck, als wenn diese Zellenbildung desto rascher und lebhafter auftritt, je kleiner das Thier und je unbedeutender die Wunde ist. Weiter scheinen stärkere Blutungen und grössere Vorfälle, sowie durch Anämie resp. Gewebszerstörung bedingte ausgedehnte Nekrosen den Proliferationsprocess zeitlich aufuhalten. Durch das Verschwinden der Leukocyten ist die Beobachtung der einzelnen Zellarten bedeutend erleichtert. Eine grosse Anzahl der jungen Granulationszellen trägt sowohl körniges, wie spiessiges Pigment. Wenn auch der grössere Theil des ersteren auf Blutpigment zurückgeführt werden 
Exp. Untersuch. über die Heilungsvorgänge von Lederhautwunden, 307

muss, so sind doch die intensiv dunkel gefärbten, fast schwarzen Körner kaum anders als früherer Inhalt der degenerirten chorioidealen Stromazellen, resp. der iibrigen pigmenthaltigen Zellen zu deuten ${ }^{1}$ ). Das spiessige Pigment kann nur von den Retinaepithelien stammen. Dieses letatere Pigment ist fast ausschliesslich in den Granulationszellen auffindbar, und es gelingt kaum, ausserhalb der Netzhaut isolirte Pigmentsplitterchen zu sehen. Nur dann, wenn die Netzhautabhebung gering war und die Degeneration der Stäbchen und Zapfen spät einsetzte, gelangten die zertrümmerten resp. in toto desquamirten Pigmentepithelzellen recht zahlreich in den Wundcanal, und unter diesen Umständen ereignete es sich häufiger als sonst, die Pigmentsplitterchen nach dem Zelltode in grosser'Dichte neben den Wundrändern zu finden. Besonders prägnant zeigte' sich diese Erscheinung, wenn die Leukocyteninfiltration des Keimgewebes durch das Abpräpariren des benachbarten episkleralen Gewebes und durch die Abtragung eines Aderhautprolapses zeitlich und numerisch ungünstig beeinflusst wurde. Ausser dem Pigment zeigen die Granulationszellen, frisch untersucht, in ihrem Protoplasma zahlreiche Fetttröpfchen, sowie glänzende unregelmässige kleine Bröckelchen und Krümelchen. Letztere befinden sich hauptsächlich in den innerhalb der Sklera gebildeten Proliferationszellen. Nach der Färbung erkennt man in denselben sehr oft kleine unregelmässige Chromatinklümpchen ausserhalb ibres eigenen blasigen und schwächer gefärbten Kernes.

Durch Nikiforoff ${ }^{2}$ ) u. A. ist es bekannt, dass die Derivate der degenerirten Zellelemente auf dem Wege der Phagocytose von den neugebildeten Keimzellen aufgenommen werden. Es wird daher der scheinbare Widerspruch, dass

1) Die Eisenreaction fiel hier ausserdem negativ aus.

2) Nikiforoff, Untersuchungen über den Bau und die Entwickelung des Granulationsgewebes. Ziegler's, Beitr. Bd, 8, S. 400. 
die neugebildeten Zellen - welche später zwar eine morphologische Umformung, aber keine pathologische Degeneration erfabren - Fetttropfen enthalten, am unverfänglichsten zur Lösung gebracht werden, wenn man diese Fetttropfen für das Entartungsproduct präformirter Grundelemente und der Leukocyten bält. Die glänzenden Bröckelchen sind wahrscheinlich als die übriggebliebenen Partikelchen der Nervenzellen aufzufassen; eine specifische Färbung glückte mir nicht. Die intensiv gefärbten Chromatinklümpchen sind als Reste von Leukocytenkernen zu deuten. Die Herkunft des Pigmentes bedarf keines weiteren Commentars.

Degenerative Processe sind ausser im Tapetum lucidum nur noch in der Netzhaut nachweisbar, wo die Pigmentepithelien, die Körnerschichten und besonders die grossen Ganglienzellen in weiterer Ausdehnung zu Grunde gegangen sind, so dass die Müller'schen Fasern einen Säulengang zu bilden scheinen (Fig. 3, 5). Am wenigsten verändert sind die Zapfen und Stäbchen, doch ist auch ihre Anzahl bedeutend verringert (Fig. 3, 5, 8).

\section{Epikrise.}

Diese Beschreibung entspricht dem mikroskopischen Bilde am Ende des zweiten und im Laufe des dritten Tages. Ein fortschreitender Gewebsuntergang ist nur noch an den Zellen des Tapetum lucidum, sowie an denen der Retina zu sehen. Dagegen beginnen überall die Reparationsvorgänge. Als weitere Erscheinung ist die mannigfaltige Phagocytose der jungen Granulationszellen anzusprechen, welche durch die Aufnahme verschiedenartigen Pigmentes (Fig. $2 \mathrm{~d}, 3 \mathrm{i}, 6 \mathrm{~d}, \mathrm{f}, 7 \mathrm{f}, 8 \mathrm{c}$ ) und der differentesten Zell- und Kerntrümmer dem histologischen Bilde ein ausserordentlich wechselndes Colorit verleiht. 
Exp. Untersuch, über die Heilungsvorgănge von Lederhautwunden. 309

Weiterer mikroskopischer Befund.

Die neuen Bildungszellen sind meistens rund oder oval, zuweilen auch stern- oder birnförmig (Fig. $2 \mathrm{c}, \mathrm{d}, \mathrm{e}$ ). Das Proliferationsvermögen der Augenhäute ist nicht nur untereinander ganz verschieden, sondern auch bei den einzelnen Geweben, je nach Ort und Lage der Verletzung, grossen Schwankungen unterworfen.

An der Retina nehmen die Müller'schen Stützfasern zu. Thr inneres Ende, sowie der Hauptstamm wird breiter. Die vorher als mehr oder weniger zarte Reiserchen erkennbaren Fortsätze sind verdickt. Dieselben überwölben entweder die leeren Nischen der zu Grunde gegangenen Ganglienzellen, oder streben als selbständige Pfeiler nach aussen, wobei sie sich häufig zwischen die Nervenzellen und Pigmentepithelien einzwängen (Fig. 5 u. 8). In der Nervenfaserschicht sind die Zellen der Adventition deutlich vermehrt, doch reicht ihre Wucherung zu einer Mitbetheiligung an der Bildung des Keimgewebes nicht aus (Fig. 3 u. 5). Die Neubildung des Gliagewebes ist gering. Es treten allerdings blasige Kerne auf, welche grösser sind wie die Kerne des präformirten Gliagewebes und kleiner wie die der Adventitiazellen, doch halten dieselben sich numerisch in engen Grenzen. Nur in den Markflügeln der Kaninchen kann man von einer Proliferation reden. Daselbst ist ausgesprochene Kern- und Faservermehrung zu beobachten, doch scheinen auch hier die Endothelien der Capillaren, sowie die Adventitiazellen dio Hauptmasse zu liefern (Fig. 5). Während bei den anderen Thierarten die Netzhaut höchstens nur soviel Material liefert, als zur Herstellung einer Vereinigung mit dem übrigen Keimgewebe nöthig ist, wobei sie ihre anatomischen Grenzen ängstlich respectirt, gehen bei geeignet gelegten Schnitten, von den Markflügeln der Kaninchen zell- und gefässreiche Stränge aus, um sich direct am Aufbau des neuen Gewebes zu betheiligen und auch ihrerseits durch active Wucherung 
mit dem von den übrigen Häuten neugebildeten Gewebe in Verbindung und sogar in Concurrenz zu treten (Fig. 5).

Seitens der Aderhaut ist die Schicht der grossen Gefässe am stärksten betheiligt durch die Lieferung mannigfaltig gestalteter Bildungszellen, welche der Wunde zustreben, um den retinalen und besonders den skleralen Schnittrand zu umsäumen (Fig. 2, 3, 5, 6, 8). In denjenigen Fällen, wo die partielle Abhebung der Netzhaut sich mit einer Retraction der Aderhaut combinirte und wo in kurzer Zeit die nervösen Zellen and mit ihnen die Pigmentepithelien in grösserer Ausdehnung zu Grunde gerichtet wurden, durchbricht die Aderhautwucherung nicht selten die Limitans, um direct von hinten in die Retina einzudringen, obne sie vorher vom Wundrande aus zu umfassen.

An der Suprachorioidea ist das durch den Eingriff entblösste Fasernetz wieder mit neuem Endothel bekleidet, welches zum Theil völlig spindelig ist, und länglich gestaltete Kerne enthält. Auch scheint diesen Zellen ein geringer Antheil an der Bildung des neuen Gewebes zugeschrieben werden zu müssen, zumal ein directes Thätigkeitszeichen in der Form der Karyokinese auffindbar ist (Fig. 2f). Die neugebildeten Zellen lassen sich in der Continuität als directe Fortsetzung der erhalten gebliebenen Endothelien verfolgen, weil sie sich nur allmählich dem Wundrande nähern, und nach seiner Ueberschreitung auf dem Querschnitt einen den Endothelien homologen Zellcharakter bewahren (Fig. 6 g, 7 Sup., 8 Sup.).

Die Sklera selbst zeigt in der Wundnähe alle Uebergangsstadien der indirecten Zelltheilung. In den Saftbahnen befinden sich reichliche Karyomitosen und junge Zellen, welche oft zu mehreren neben und hinter einander liegen (Fig. 2, 3, 4, 5, 6, 7, 8). Allerdings betheiligt sich die Lederhaut an der Bildung des den Wundcanal ausfüllenden Granulationsgewebes nicht. Sie producirt zwar viele neue Zellen, aber schickt keine einzige in den Wundcanal, 
Exp. Untersuch. über die Heilungsvorgänge von Lederhautwunden. 311

und verhält sich auch dann exclusiv, wenn ihr allein die Aufgaben der Heilung zufallen sollten, d. h. in allen den Fällen, wo sie von den anliegenden Häuten entblösst ist. Aber gerade dann zeigt die Lederhaut ihre Ohnmacht, sowie ihre Abhängigkeit rom Nachbargewebe. Ausser einer merklichen Verbreiterung der nekrotischen Randzone entwickelt sich unter solchen Umständen, die durch Karyomitose eingeleitete Zellproliferation viel später und in langsamerem Tempo, so dass auch jetzt eine Uebereinstimmung mit den gewöhnlicheren Verhältnissen nicht vermisst wird. Die intrasklerale Wucherung ist in ihrem Beginn völlig unregelmässig (Fig. 2) und erst allmählich legen sich die neuen Zellen den Fibrillenbündeln an (Fig. 3, 4, 5, $6,7,8)$.

Der Ciliarkörper zeigt bei seiner Verletzung eine Abnahme der Muskel- und eine Zunahme der Bindegewebselemente. Diese Erscheinungen sind so auffällig, dass sie sich schon bei schwacher Vergrösserung durch den Schwund des angrenzenden Muskelbündel und eine Verbreiterung der bindegewebigen Septen deutlich von einander unterscheiden lassen (Fig. $7 \mathrm{a}, \mathrm{b}, \mathrm{c}, \mathrm{d}$ ).

Am schönsten markirt sich bei allen Thieren die Neubildung des episkleralen Gewebes. Auf den ersten Blick erkennt man langgestreckte, etagenartig angeordnete Zellindividuen, welche leistenförmig die sklerale Aussenfläche bedecken und senkrecht zum Wundrande ziehen (Fig. 2, 7 Ep., 8 Ep.). Eine Differenz zwischen der Wucherung der Bindegewebszellen und der Endothelien ist allerdings nur ausnahmsweise zu finden, da die Zellcontouren und die Karyomitosen dieser beiden Zellarten nur dann mit Sicherheit von einander unterschieden werden können, wenn die Zugehörigkeit des Protoplasma und der Kerntheilungsfiguren in scharfer und klarer Weise ausgeprägt ist: eine Erscheinung, welche bei der dichten Lagerung und der ausgedehnten Zellproduction nur selten constatirt werden kann. 
Aus den Interstitien der willkürlichen Muskulatur werden gleichfalls mannigfache Zellzuige zum $W$ undcanal zugesandt; doch betheiligt sich der hintere Abschnitt der einzelnen Muskel nur wenig im Verhältnisse zum vorderen Muskelende (Fig. $3 \mathrm{M}$ ).

Nicht unerwähnt möchte ich lassen, dass die Adventitiazellen der angrenzenden Gefässe, sowie die Zellen des benachbarten Perineurium sich in ausgedehnter Weise am Proliferationsprocess betheiligen und selbst innerhalb der sonst ziemlich indifferenten Lederhaut eine Zellvermehrung erkennen lassen. Diese Bemerkung ergiebt sich theilweise von selbst, zumal wir gesehen haben, dass das Hauptcontingent der chorioidealen und retinalen Bildungszellen von den Gefässadventitien geliefert wird.

Das neue Keimgewebe setzt sich - wie soeben auseinandergesetzt - aus den Producten aller Häute zusammen. Es empfiehlt sich, aus Bequemlichkeitsrïcksichten, den Antheil der einzelnen Bestandtheile an der Bildung summarisch zu behandeln, und das von den äusseren Augenhäuten gelieferte Bildungsmaterial als äusseres, sowie die aus dem Augeninnern stammenden Zellzüge als inneres Keimgewebe zu bezeichnen.

Bei der Verheilung einer uncomplicirten Wunde im vorderen Augenabschnitt, ohne wesentliche Lagerverschiebung, und mit Schonung der äusseren Augenmuskeln sowie des Ciliarkörpers, betheiligen sich inneres und äusseres Keimgewebe fast gleichmässig. Dieselben treffen sich in der Wundmitte. Die Führungslinie der Keimgewebe bilden die Wundränder. An diese lagern sich die jungen Zellen direct an, um den Canal zu durchziehen. Die Abkömmlinge des inneren und äusseren Zelllagers folgen im Allgemeinen einer geraden Linie; desgleichen steht die Längsaxe ihrer Kerne und des Protoplasmas in der Regel parallel dem Zellzuge. Aus diesen Gründen ist es nach ihrer Begegnung meist unmöglich, eine Scheidung vorzunehmen, 
Exp. Untersuch. über die Heilungsvorgänge von Lederhautwunden. 313

zumal die Verschmelzung eine sehr innige ist, und morphologische Unterscheidungsmerkmale fehlen (Fig. 3, 7, 8).

Man könnte ja allenfalls das von den Granulationszellen aufgenommene Pigment der zerstörten Retinaepithelzellen und der chorioidealen Stromazellen zur Diagnose der Zellabstammung verwerthen; doch lässt auch diese Argumentation wegen des verschiedenartigsten Pigmenttransportes im Stich. Dasselbe konnte entweder durch den in den ersten Tagen vermehrten Säftestrom in den Wundcanal gelangen, oder durch die Leukocyten weiter verschleppt werden; so dass eine Phagocytose an allen Orten des neuen Keimgewebes möglich wurde. Allerdings findet man in der inneren Wundhälfte die pigmenthaltigen Granulationszellen viel zahlreicher vor, wie in der äusseren; doch liegt dies in der Natur der Sache, weil das Pigment aus dem Augeninnern stammt und mit der Entfernung von seinem ursprünglichen Sitz abnehmen muss. Das Blutpigment, sowie das übrige körnige Organpigment ist für die Diagnose des Zellursprungs gleichfalls werthlos, weil Ersteres iuberall in der Wundumgebung von den Keimzellen aufgenommen werden konnte, und Letzteres sich regelmässig in der Sklera und den äusseren Geweben auch dann nachweisen liess, wenn es in den Adventitiazellen der Aderhaut sichtbar war.

Fine stärkere Entwicklung des äusseren Keimgewebes tritt hervor bei Verletzungen des Ciliarkörpers (Fig. 7), und besonders bei gleichzeitiger Verwundung der äusseren Augenmuskeln; denn der Ciliarkörper leistet bei der Wundheilung nur vorbereitende Dienste, weil er sich ausschliesslich auf die Lieferung des Zellmaterials beschränkt, welches nöthig ist, um eine Verlöthung mit dem aus dem episkleralen Gewebe stammenden Bildungszellen zu Stande zu bringen. Dagegen sekundirt das interstitielle Gewebe der quergestreiften Muskulatur den übrigen episkleralen Schichten stets in so ausreichendem Maasse (Fig. $3 \mathrm{M}$ ), dass es bei seiner eigenen Verletzung sogar im Stande ist, der Aderhaut- 
wucherung allein die Waage zu halten. Ein Uebergewicht des inneren Keimgewebes ist bedingt durch Entwicklung und grössere Betheiligung der Suprachorioidea, wie dies am Besten bei Katzen und Hunden in die Augen fällt. Es entstehen hier dieselben Zellformationen, wie sie S. 310 beschrieben sind; nur mit dem Unterschiede, dass - ausser der Regeneration für die functionsfähig gewordenen Endothelien - sich nicht einige wenige neugebildete Zellexemplare am Aufbau des Keimgewebes betheiligen, sondern durch die Production mehrerer Zelllagen dichte Kernreihen gebildet werden. Allerdings besteht hier oft die Gefahr der Täuschung. Bei Dehiscenz der suprachorioidealen Lamellen zweigen sich von den vorbeipassirenden, polymorphen Granulationszellen der übrigen Gewebe gerne einige $a b$, um die erweiterten Lymphräume der Suprachorioidea auszufüllen, wobei sie durch die platten und meist länglichen Endothelzellen in einfacher oder vermehrter glatter Zelllage eingefasst werden. Stets bleibt die Aderhaut in der hinteren Bulbushälfte der Hauptlieferant der jungen Zellen, denn die Tenon'sche Kapsel producirt im günstigen Falle nur eine verhältnissmässig schwach entwickelte Zellschicht, welche zwar die äusseren Wundwinkel bedeckt, aber zur gegenseitigen Vereinigung nicht ausreicht. Und selbst in der Nähe des Ansatzes des Musc. retractor. bulbi genügt die Hilfe seines bindegewebigen Antheiles in der Regel nicht, um mit der Aderhaut gleichen Schritt zu halten (Fig. 8Ep). Die Stromapigmentzellen scheinen, wie iberall, so auch hier, inactiv zu bleiben. Eine selbständige Netzhautwucherung beschränkt sich - wie schon oben erwähnt - allein auf die Markflügel der Kaninchen.

Wie Seite 310 auseinandergesetzt, betheiligt sich die Sklera an der Bildung des die Wunde ausfüllenden Granulationsgewebes nicht. Stets ist die Wucherung des jungen Keimgewebes so ausgiebig, dass das neugebildete intrasklerale Zellmaterial nur in seltenen Fällen die fremden. 
Exp. Untersuch. über die Heilungsvorgänge von Lederhautwunden. 315

Granulationszellen direct an der Wundgrenze zu empfangen vermag, weil es in der Regel durch die von aussen kommenden Keimzellen in den eigenen Saftlïcken überrascht wird. Die rom Keimgewebe sich abzweigenden und in die Sklera einwandernden Zellen biegen zunächst nur mit einem Theil ihrer Massen in die Saftlücken ein. Später auftretende schieben sich dachziegelförmig und perlschnurartig vor (Fig. $31,6 \mathrm{~b}, 7,8)$. Bei ausgiebiger Zellproliferation sind auch in der Sklera die Uebergänge bald verwischt, und es lässt sich auch hier der Ursprung der Zellen bis zur gegenseitigen Berührung nur durch Verfolgen der Neubildungsvorgänge, und nicht durch die Zellart erkennen. Unter gewöhnlichen Bedingungen bilden die jungen, in den Saftlücken sichtbaren Zellen am dritten bis fünften Tage ein dichtes Continuum, mit der Längsaxe senkrecht zum Wundcanal (Fig. 3, 5, 4, 6, 7, 8).

$\mathrm{Zu}$ dieser Zeit sind auch schon reichlich neue Gefässe entwickelt. Dieselben haben eine dünne Wand, sowie ein weites, meist mit rothen Blutkörperchen vollgepfropftes Lumen. Die grösste Anzahl stammt aus dem vorderen Abschnitt des episkleralen Gewebes (Fig. 7) und aus der Choriocapillaris (Fig. 3 h). Die anderen Gewebe betheiligen sich nur wenig oder gar nicht an der Gefässentwickelung. Eine Gefässneubildung aus dem Ciliarkörper habe ich mit Bestimmtheit nicht beobachten können; desgleichen scheinen die Retinagefässe keine jungen Capillaren zu liefern. Nur in den Markflügeln der Kaninchennetzhaut, wo die Gliawucherung recht bedeutend sein kann, ist auch Gefässneubildung vorhanden und mitunter ausserordentlich deutlich ausgeprägt (Fig. 5 k). Das interstitielle Bindegegewebe der quergestreiften Muskulatur betheiligt sich nur in den vorderen Muskelabschnitten gleichfalls an der Gefässneubildung, doch rangirt es erst in zweiter Linie. Die Sklera selbst scheint auch in dieser Beziehung völlig inactiv. Es ist mir selbst bei Thieren mit weniger gefässarmer 
Lederhaut nie gelungen, ein neugebildetes Gefäss zu entdecken. Mitunter findet man allerdings ein im Wundcanal endendes, blutkörperchenhaltiges Skleralgefäss, doch ist dasselbe stets als ein präformirtes zu erkennen. Es ist ja nicht ausgeschlossen, dass ein solches Gefäss als Zeitbahn benutzt wird, aber es lässt sich auch hierüber ein bestimmtes Urtheil nicht fällen.

Beobachtet man die Wunde nach ihrer zelligen Umsäumung von der Fläche, so zeigt sich unter normalen Verhältnissen ihr äusserer Rand fast gleichmässig durch Granulationszellen eingefasst. An den Wundlippen und Wundwinkeln biegen die Zellen fast rechtwinklig in den Canal hinein (Fig. 3, 4, 5, 6, 7, 8), so dass hier nur Querschnitte sichtbar sind. Kurz darauf spannt sich eine Zelldecke durch oder über die Wunde, welche entweder an der engsten Stelle oder am Orte der stärksten Gewebswucherung gebildet wird.

Beim Zusammentreffen beider Momente wird die Wundöffnung in wenigen Stunden uiberbrïckt, doch nimmt selbst unter ungünstigen Verhältnissen der zellige Verschluss nur kurze Zeit in Anspruch. Ist diese Brücke fertig, so beginnt die weitere Ausfüllung des Wundcanals mit Granulationszellen. Stets reihen sich die jungen Zellen appositionell dem schon fertigen Keimgewebe an, indem sie den Hohlraum der Wunde flächenhaft auskleiden. Ein regelloses Hineinwuchern in die Wunde konnte ich nie bemerken. Die Ausfüllung und Verengerung der Wunde erreicht unter normalen Bedingungen ihren Abschluss im Niveau der Suprachorioidea resp. Aderhaut und des episkleralen Gewebes (Fig. 7 u. 8).

Unter günstigen Umständen kann die vollständige Ausfüllung einer Wunde schon am dritten Tage beendigt sein, doch nimmt sie für gewöhnlich vier bis sieben Tage in Anspruch.

Von einem Gewebsschwunde ist kaum mehr etwas zu 
Exp. Untersuch. über die Heillungsvorgänge von Lederhautwunden. 317

sehen. Nur die Zellen der Ganglienzellenschicht degeneriren auch jetzt noch in grosser Ausdehnung nach allen Richtungen (Fig. 5, 8), während die Körmerschichten, die Zapfen and Stäbchen, sowie die Pigmentepithelzellen nur noch in der unmittelbaren Wundnähe zu Grunde gegangen sind. Neubildungsprocesse habe ich an diesen Zellen nicht gesehen.

\section{Epikrise.}

Die Reparationsbestrebungen sind nach den obigen Auseinandersetzungen in allen Geweben vorhanden, doch unterscheiden sich die einzelnen Wucherungsvorgänge in wesentlicher Hinsicht. Das numerische Verhältniss der neuen Bildungszellen entspricht der formativen Thätigkeit einer ersatzfähigen Matrix und diese ist, wie sich Birch-Hirschfeld ${ }^{1}$ ) zutreffend ausdrückt, „in der Regel auf das Innigste mit der Neubildung von Blutgefässen verbunden, da namentlich eine Regeneration des gefässhaltigen Bindegewebes und auch eine Ausfüllung von Substanzverlusten in anderen Geweben durch solches nur unter der Voraussetzung gleichzeitiger Neubildung von Blutgefässen stattfindet. Diese Neubildung spielt eine wichtige Rolle bei der Wundheilung, wenn die directe Vereinigung der getrennten Gewebe verhindert ist, und die Ausfüllung der entstandenen Lücke durch secundäres Hineinwuchern von gefässhaltigem Bindegewebe ausgefüllt wird."

Da die Proliferationsvorgänge der Lederhaut zu gleicher Zeit mit denen der Nachbargewebe einsetzen und sich in gleicher Schnelligkeit abspielen, so kann den Sklerazellen der Vorwurf einer schwach entwickelten oder abgeschwächten Lebenskraft und Fortpflanzungsfähigkeit nichtgemachtwerden. Es ist vielmehr die Ursache des spärlichen ProliferationsS. 128.

1) Birch-Hirsehfeld, Grundriss der Allgem. Pathologie 1892, 
vermögens der Lederhaut in anderen Momenten zu suchen. Mögen dieselben auch zum grossen Theil auf der relativ geringen Anzahl der präformirten Sklerazellen beruhen, so ist doch die Gefässarmuth der Lederhaut als ausschlaggebender Factor mit in Rechnung zu ziehen, zumal es auch nicht ein einziges Mal gelang, ein neugebildetes Skleralgefäss zu entdecken. Mit der Birch-Hirschfeld'schen Anschanung correspondirt weiter die dominirende Zellwucherung der Aderhaut in der hinteren Augenhälfte, wo die Tenon'sche Kapsel zwar zellreicher, aber blutärmer als vorne ist (Fig. 6, 8). Desgleichen kann der Unterschied der Proliferationsvorgänge am episkleralen Gewebe des vorderen und hinteren Bulbusabschnittes in unmittelbare Beziehung zu dem Contrast gesetzt werden, welcher durch die ungleiche Gefässvertheilung an diesen beiden Augentheilen geschaffen wird. Schliesslich wird es sogar verständlich, dass das bei den meisten Wundheilungen proliferativ nux spärlich in Erscheinung tretende Neurogliagewebe in den gefässreichen Markflügeln der Kaninchen einen gewissen Grad ron Activität erreicht (Fig. 5). Allerdings bleibt die Reservirtheit des verletzten Ciliarkörpers unerklärt, doch wissen wir aus dem analogen Verhalten der Muskelwunden im Digestionstractus und im Uterus, dass das glatte Muskelgewebe mit seinen bindegewebigen Interstitien wenig Neigung zu ausgiebiger Proliferation zeigt und sich in der Regel auf eine feste Verwachsung seiner Wundflächen beschränkt.

\section{Anfang der zweiten Woche bis Fnde des vierten Monats.}

Grob anatomischer und klinischer Befund.

Die Wunde bleibt an der Aussenfläche der Sklera noch lange sichtbar, weil sie sich als bläulicher Spalt lebhaft von der weissen Lederhaut abhebt. Ophthalmoskopisch ist zu erkennen, wie der Glaskörper allmählich durchsich- 
Exp. Untersuch, über die Heilungsvorgänge von Lederhautwunden. 319

tiger wird. Die verletzte Stelle zeigt ein weisses, zum Theil schwarzgesprenkeltes Aussehen, welches besonders bei Thieren mit einem Tapetum lucidum sehr in die Augen fällt. Die Netzhaut ist in der Wundumgebung getrübt. Wurden durch die Verletzung die Retinagefässe durchschnitten, so ist das periphere Gefässende nicht mehr auffindbar.

\section{Mikroskopischer Befund.}

Sowie das Keimgewebe die Wunde geschlossen hat, verändert es seine Organisation. Die Zellen und Kerne des eingelagerten Gewebes werden spindelig und legen sich in parallele Reihen, so dass zwischen ihnen und den präformirten Sklerazellen morphologische und topographische Aehnlichkeiten zu Tage treten. Zugleich werden reichliche Fibrillen gebildet, welche in der ersten Zeit noch regellos einander durchkreuzen, aber schon nach drei Wochen (Fig. 7) in welliger, parallel-faseriger Anordnung die Sklerafragmente verbinden. Diese Veränderungen gehen nur langsam vor sich, und erst gegen Ende des zweiten Monats prävalirt die fibrilläre Componente vor der zelligen (Fig. 8). Von dieser Zeit an bleibt nur noch die grössere Kernanzahl ein sicheres Erkennungszeichen des neugebildeten Gewebes gegenüber dem fast homogenen Mattenwerk der Lederhaut.

In demselben Verhältniss, wie die Transformation der Granulationszellen in die Fibroblasten- und die definitive Fibrillenbildung vor sich geht, und wie aus dem allgemeinen Zelldurcheinander durch Umlagerung und Umordnung sich in gleicher Fläche mit dem Skleragewebe Spindelzellen und Fibrillen entwickeln, collabiren die neugebildeten Gefässschlingen (Fig. $7 \mathrm{~g}$ ), um zuletzt völlig zu verschwinden (Fig. 8). Nur sehr selten restirt noch hier und da ein kleines Gefässchen als einziger Ueberrest der früheren localen Circulationsvermehrung. Die neugebildeten Fibrillen verfilzen sich auf das allerinnigste miteinander, mit den 
präexistirenden skleralen Bündeln, den Lamellen der Suprachorioidea und dem Zellgewebe der Tenon'schen Kapsel (Fig. 7 u. 8). Am auffallendsten ist der Uebergang an der Schicht der grossen Aderhautgefässe und der Choriocapillaris (Fig. 8), weil hier die zahlreichen Zellen und die vielen Gefässlumina lebhaft mit den fibrillären Faserzügen contrastiren. Die reichliche Zellanhäufung in den skleralen Saftlïcken, von denen, wie wir gesehen haben, eine grosse Anzahl auf das Conto des von aussen eindringenden Keimgewebes zu setzen ist, hat an den Wundrändern eine so feste und gleichmässige fibrilläre Verbindung hergestellt, dass eine Scheidung zwischen alten und neuen Fibrillen in hohem Maasse erschwert ist (Fig. 8). Durch die Erzeugung und Einlagerung einer homogenen Interfascicularsubstanz wird dies Ineinandergreifen der einzelnen Fibrillen noch mehr befestigt und verstärkt. Eine Neubildung von Fibrillen aus Fibrillen ist mit Sicherheit zu verneinen, vielmehr gelten auch hier dieselben Grundsätze, wie sie bei der Heilung von Sehnenwunden formulirt worden sind, wenn von einer „,ingerartigen Verschränkung und einer Juxtaposition“1) der Fibrillen geredet wurde.

\section{Epikrise.}

Die histologische Gleichartigkeit der Lederhaut und des Ersatzgewebes, welches aus dem Protoplasma der Keimzellen seinen Ursprung nimmt, könnte die Annahme gerechtfertigt erscheinen lassen, die Heilung der Lederhautwunde als einen vollständigen regenerativen Vorgang aufzufassen. Die relative Inactivität der Lederhaut und die dominirende formative Thätigkeit der Nachbarhäute sind allerdings schon Gründe genug, um diese Frage mit Bestimmtheit zu verneinen. Als eine vollständige Regeneration

1) Beltzow, Entwicklung und Regeneration der Sehnen. Arch. f. mikr. Anat. Bd. 22. S. 737 . 
Exp. Untersuch. über die Heilungsvorgänge von Lederhautwunden. 321

wird man diesen Process nur dann bezeichnen können, wenn er zur Herstellung des stabilen Gleichgewichtes ausser der anatomischen Ebenbürtigkeit eine physiologische Gleichwerthigkeit zu schaffen vermag. Trotz der grossen Aehnlichkeit zwischen dem alten skleralen und neuen intraskleralen Gewebe vermisst man die typische Anwendung der Saftlücken. Die Fibrillen liegen in so dichten, compacten Reihen, dass es niemals gelingt, einzelne Bündelgruppen voneinander abzugrenzen, wie es in der Lederhaut mit Leichtigkeit durchgeführt werden kann (Fig. 8). Es wird daher die Charakterisirung des interponirten Gewebes auf das eines Ersatzgewebes reducirt werden müssen, welches wegen seines Zellreichthums, sowie wegen seiner fibrillären, welligen Anordnung dem skleralen Gewebe morphologisch analog zu stellen ist, aber wegen des Mangels eines typischen Saftcanalsystems als ein physiologisch minderwerthiges aufgefasst werden muss. Andererseits kann ich die von den angeführten Autoren benutzte Bezeichnung Narbengewebe nur für die zellig-fibrilläre Verbindung der Wundräncler an den inneren Augenhäuten und dem episkleralen Gewebe acceptiren, während ich für den intraskleralen Antheil den Ausdruck Ersatzgewebe vorziehen möchte.

\section{Weiterer mikroskopischer Befund.}

Die Wundränder der Netzhaut verwachsen niemals direct miteinander, und selbst in den Markflügeln der $\mathrm{Ka}$ ninchen wird die retinale Lücke zum Theil durch die Zellproliferation anderer Häute ausgefuillt. Bei regelrechter Heilung bleibt stets eine Diastase zurück. Dieselbe documentirt im allgemeinen das frühere Klaffen der Wunde, da die Wundränder der Netzhaut wegen ihrer schwach entwickelten elastischen Elemente meistens in situ verharren, ohne sich merklich vom Niveau der skleralen Wundfläche zurückzuziehen. Der Defect in der Limitans interna 
schliesst sich meist scharf gegen den Glaskörper ab. Mitunter erkennt man auf dem Innern der geheilten Wunde eine Reihe platter Zellen, welche als die Fortsetzung der Limitansendothelien angesehen werden können (Fig. 8 d). Isolationspräparate sind mir allerdings nicht geglïckt, doch halte ich die Restitution von endothelialen Elementen an dieser Stelle für äusserst wahrscheinlich, zumal nach der Analogie auch dann Defecte mit Endothelien wieder ausgefiullt werden, wenn ihre Basalmembran eine dauernde Schädigung erfahren hat, resp. theilweise zerstört ist. Bei ungestörtem Heilungsverlauf findet man ein allmähliches Zusammenrücken der arkadenartig angeordneten Müllerschen Stützfasern. Ausserdem werden in Folge der Neuroglianeubildung die durch Nervenzellennekrose entstandenen Lüicken verengt. Da die Wucherung des Stützgewebes nicht ausreicht, um die zu Grunde gegangenen Nervenelemente territorial vollkommen zu ersetzen (Fig. 8b), so entsteht eine Gewebsverdichtung mit Volumenverminderung, welche die retinalen Wundränder als zugeschärft erscheinen lässt (Fig. 8). Diese Verschmälerung wird zuweilen dadurch ausgeglichen, dass die Verbindung des retinalen Stitzgewebes mit den Proliferationszellen der Aderhaut eine sehr ausgedehnte sein kann; doch pflegt im allgemeinen das von der Aderhaut gelieferte und in die Netzhaut eindringende Keimgewebe nur dann eine grössere Mächtigkeit zu zeigen, wenn die Aderhaut sich stark retrahirte, sowie wenn die Degeneration und Zerstörung der Nervenzellen die gewöhnlichen Grenzen überschritt.

Es können hier nur diejenigen Veränderungen der Retina berücksichtigt werden, welche sich unmittelbar am Wundcanal abspielen und welche für das Verständniss des Heilungsvorganges nothwendig sind. Da sich jedoch bei der Untersuchung mancherlei werthvolle und interessante Befunde ergeben haben, so werden dieselben am Ende der Arbeit eine nähere ausführliche Schilderung erfahren und 
Exp. Untersuch. über die Heilungsworgänge von Lederhautwunden. 323

mit der Gesammtepikrise der retinalen Veränderungen gemeinschaftlich abgehandelt werden.

Eine active Betheiligung des Ciliarmuskels ist mit positiver Bestimmtheit nicht zu constatiren, vielmehr entsteht eine deutliche Narbe von derbem, fast straffem Charakter, an deren Umgebung sich eine Gewebsabnahme nachweisen lässt, welche zum Theil durch die Schrumpfung der Narbe selbst zu Stande kommt, zum Theil durch die den operativen Eingriff begleitende Zerstörung und Degeneration der benachbarten Muskelelemente hervorgebracht ist (Fig. 7c, d). Desgleichen konnte in den übrigen Theilen des Ciliarkörpers niemals eine vicarïrende Muskelneubildung aufgefunden werden, welche die durch den Defect bedingte Herabsetzung der Leistungfähigkeit - in Form einer compensatorischen Hypertrophie - auszugleichen im Stande gewesen wäre.

Beim Verheilen einer Ciliarkörperwunde begegnet man einer merkwürdigen Erscheinung, auf welche schon vor langer Zeit Sattler ${ }^{1}$ ) aufmerksam gemacht hat. Die Epithelien der pars ciliaris retinae nehmen in der Wundnähe eine lange, spindelige Form an und sind selbst bei starker Vergrösserung kaum von Spindelzellen zu unterscheiden. Wenn sie sich auch am Heilungsprocesse nicht direct zu betheiligen scheinen, so wuchern sie doch häufig so zahlreich in den Glaskörper hinein, dass ihr specieller Zellcharakter verloren geht und man Spindelzellen vor sich zu haben glaubt. Es ist dies Phänomen ein histologisches Unicum, welches ohne Parallele dasteht und nur in theilweise Analogie mit den unter pathologischen Vorgängen spindelig veränderten Epithelzellen der vorderen Linsenkapsel zu setzen ist.

Von der Conjunctiva habe ich nur dann gesprochen,

1) Sattler, Cyclitis suppr. Abscessbild. i. Glaskörper. Atlas d. Pathol. Top. d. Auges. II. Lief,, Taf. XIII. S. 44. 
wenn ihre Mitbetheiligung mit Sicherheit constatirt werden konnte. Dieselbe beschränkt sich wahrscheinlich nur auf die Verklebung ihrer eigenen Wundränder, denn es gelang mir niemals, an ihr eine grössere formative Thätigkeit nachzuweisen. Bemerken will ich noch, dass das Epithel sich völlig regenerirt und zum Theil in mehrfacher Zelllage die durch die Wundheilung bedingten kleinen Vertiefungen und Unebenheiten auszugleichen sucht.

Unsere Untersuchungen zeigen eine erfreuliche Uebereinstimmung, mit den Angaben von E. Franke, woil er das die Sklerawunde ausfüllende Granulationsgewebe gleichfalls von den Proliferationsvorgängen der Aderhaut und des episkleralen Gewebes ableitet. Dieser gemeinsame Befund berechtigt dazu, der Frage näher zu treten, in welchem Verhältniss sich die einzelnen Zellarten dieser beiden Gewebe am Heilungsprocess betheiligen.

Im episkleralen Gewebe gelingt es wegen des dichten Maschenwerkes selbst bei dünnsten Schnitten allerdings nur selten, die fixen Bindegewebszellen von den Endothelien zu unterscheiden, zumal die bei der Zelltheilung zu Stande kommende Kernvergrösserung so raumbeengend wirkt, dass die Zellgrenzen nicht zu erkennen sind. Dagegen kann an der Suprachorioidea auf dem Querschnitt die directe Verbindung des übrigen Keimgewebes mit den Producten der endothelialen Matrix häufig genug nachgewiesen werden, weil der neugebildete aus länglichen und platten Zellen bestehende Zellfaden in directem Zusammenhange mit den präformirten Endothelien steht und sich in den ersten Entwicklungsphasen deutlich von den übrigen polymorphen Keimzellen abgrenzen lässt (Fig. $6 \mathrm{~g}$ ). Nach der Umordnung in die Spindelform verwischt sich allerdings anch hier der Unterschied (Fig. 7, 8 Sup.). 
Exp. Untersuch. über die Heilungsvorgänge von Lederhautwunden. 325

Epikrise.

Die reichhaltige Betheiligung der Endothelien am Aufbau des Keimgewebes ist immerhin auffällig; doch wird dieselbe verständlich, wenn man bedenkt, dass die Endothelien und Bindegewebszellen von gleicher Genese sind. Endothelien sind im Grunde nur umgebildete Bindegewebszellen, welche - im Gegensatz zu den in kleineren Gewebsspalten, z. B. den intraskleralen Saftlücken scheinbar isolirt liegenden Zellen - in grösseren Spalten und besonders auf Binnenflächen eine continuirliche Decke bilden. Thre Anordnung, sowie ihre Bestimmung, sich auf grösseren Flächen auszubreiten, bedingen ihre morphologische Differenzirung; aber beide Zellarten haben im postembryonalen Leben die Fähigkeit, durch Wucherungsprocesse Fibroblasten und später Fibrillen zu bilden. Die Verwachsung des Ductus Botalli, und des Ligamentum teres, die Thrombus-Organisation, die Endarteriitis chronica, sowie die bindegewebige Obturation eines unterbundenen Gefässes kommt in erster Linie durch die Proliferation der Endothelzellen zu Stande. Ausserdem hat Graser ${ }^{1}$ ) durch ausschliessliche endotheliale Wucherung die Verwachsung peritonealer Blätter nachweisen können. Als letzter Grund für die endotheliale Fibroblastenbildung gelte die Annahme, dass bei der Lebercirrhose und der Niereninduration eine Bindegewebsneubildung aus den Capillarendothelien stattfinden kann.

Während ich es ron den Stroma-Pigmentzellen der Suprachorioidea unentschieden lassen muss, ob sie nur degenerative Veränderungen eingehen oder auch activ thätig sind, ist bei den Tapetumzellen jeglicher Proliferationsprocess ausgeschlossen. Durch die Untersuchungen Sattler's ${ }^{2}$ )

1) Graser, Tntersuch. über die feineren Vorgänge bei der Verwachs. peritonaler Blätter. Deutsche Zeitschr. f. Chirurgie. Bd. 27, 1888.

2) Sattler, Ueber den feineren Bau der Chorioidea des Menschen nebst Beitr. z. pathol. u. vergl. Anat. d. Aderhaut. Archiv f. Ophthalm. Bd. 22, Abth. 2, S. 54 . 
E. Krückmann.

ist bewiesen, „dass das Tapetum cellulosum lucidum bei allen Säugethieren eine mehrfache Zellschicht unter dem ersten subcapillären Endothelhäutchen darstellt". Es sind also die Tapetumzellen höher differenzirte und organisirte Endothelien, welche durch ihre physiologische Emancipirung eine Einbusse an regenerativer und formativer Thätigkeit erlitten haben; und es bestätigt sich auch hier wieder die Erfahrung, dass die functionelle Distinction der Zelle mit der Empfindlichkeit gegen Gewebsschädigungen in ein directes Verhältniss zu setzen ist.

\section{Mikroskopischer Befund der Wundstörungen.}

Es ist bis jetzt fast ausschliesslich rom regelmässigen Wundverlauf gesprochen worden, obwrohl ungünstige Abweichungen häufig genug constatirt werden konnten. Auch hier mag es uns gelingen ein Heilungsprogramm aufustellen, wenn wir unsere Beobachtungen mit den Ausführungen von Birch-Hirschfeld ${ }^{1}$ ) in Einklang bringen. Nach BirchHirschfeld „hängt der Verlauf der Wundheilung von der Ausdehnung, Form der Wunde und von der Natur der getroffenen Gewebe ab, namentlich soweit letztere die Regenerationstähigkeit bestimmt. Es ist klar, dass nach der Grösse des Substanzverlustes und der Entfernung der regenerationsfähigen Wundränder die Ansprüche an die gewebebildende Leistung vielfältige Abstufungen zeigen müssen, und es ist ferner einleuchtend, dass reine Schnittwunden in dieser Hinsicht die günstigsten Verhältnisse darbieten, doch zeigen auch die Letzteren wieder Unterschiede, je nachdem die mechanischen Verhältnisse der Umgebung einwirken."

Verbreitert sich nach Lospräparirung der Bulbushüllen resp. Abtragung eines Aderhautprolapses die nekrotische Zone der Slklera, so geht - wie wir gesehen haben - die Wundheilung langsamer und ungleichmässiger von

1) I c. S. 218. 
Exp. Untersuch. über die Heilungsrorgänge von Lederhautwunden. 327

Statten. Dazu kommt, dass die nekrotischen Spitzen der Wundränder in grösserer Ausdehnung der Resorption anheimfallen, wie unter gewöhnlichen Umständen. Durch diese vermehrte Resorption entsteht eine Vergrösserung des Defectes, welche ihrerseits wieder, ebenso wie beim Klaffen der Wunde, mit Spannungsdifferenzen einhergeht. Ausserdem scheint die Lederhaut - in Folge der sie in verschiedenen Richtungen durchflechtenden Bündel - nicht so innig und fest gewebt $\mathrm{zu}$ sein, dass sie bei Trennung ihrer Continuität in ihrer Gesammtheit zur Wirkung kommt, denn es hat oft den Anschein, dass einzelne Fibrillenbündel am Wundrande durch einen abzweigenden Faserverlauf die Spannung local zu variiren vermögen. Da eine normale Heilung nur unter gleichmässigen histomechanischen Bedingungen zu Stande kommen kann, wobei die Zugrichtung und die Spannung der Sklera bestimmend auf die Umordnung und Umlagerung der zelligen und fibrillären Elemente einwirkt, so bietet eine glatte Wunde, ohne wesentliche Diastase und Lagerverschiebungen der Fragmente, die günstigsten Heilungschancen. Hierdurch erklärt es sich, dass die subconjunctivalen und submuskulären Schnitte durch die Einengung und Beschränkung der Retraction an den skleralen Wundrändern die relativ besten Resultate lieferten, denn es ereignet sich selbst bei discret ausgeführten Schnittwunden recht oft, dass das zellig-fibrilläre Keimgewebe als Pfropf im Glaskörperraum sichtbar wird, und nicht allein die regelrechte Verheilung des inneren Wundrandes stört, sondern sogar unmöglich macht. Da die Wundränder der Aderhaut wegen ihres Reichthums an elastischen Elementen sich häufig vom Niveau der skleralen und retinalen Schnittflächen zurückziehen, so wird das Augeninnere gleichsam coulissenartig durch die Retinafragmente abgeschlossen. Da ferner durch diese Retraction die Aderhautproliferation an der unmittelbaren Umsäumung des inneren Wundrandes rerhindert wird, so 
verliert sie häufig die Fähigkeit, dem von aussen eindringenden und lebhaft wuchernden Keimgewebe hinreichend mechanischen Widerstand zu leisten. Hierdurch kann ein Hineinwuchern dieses Gewebes in den Glaskörper sehr begünstigt werden.

Derselbe Vorgang wiederholt sich, wenn durch Spannungsunterschiede die $W$ undflächen oder auch nur die inneren skleralen Faserbündel in den Glaskörper hineinragen. Durch die Verschiebung des Wundcanals nach innen wird der Heilungsprocess gleichfalls in's Augeninnere verlegt. Als weiteres Moment kommt das trichterförmige Hineinziehen des episkleralen Gewebes in Betracht, welches auf diese Weise direct in den Glaskörperraum eindringen kann. Da jedes dieser drei Momente unter Umständen zu genïgen scheint, um eine Wucherung im Bulbusinnern zu etabliren, so stellt sich bei ihrer Gesammtwirkung der Heilungsverlauf ausserordentlich ungünstig. Noch verderblicher können sich die Verbältnisse am Ciliarkörper gestalten, wo ein Antagonismus gegen das äussere Keimgewebe kaum in Frage kommt; denn nur bei den Thieren mit stark entwickelten suprachorioidealen Fasern gelingt es durch die Coalition mit den an dieser Stelle neugebildeten Endothelien eine selbständige Verbindung herzustellen, ohne dass die Dazwischenkunft des von aussen eindringenden Ersatzgewebes nothwendig wird (Fig. 7).

Im Allgemeinen kann man sagen, dass bei penetrirenden Bulbuswunden ein histologisch guter Heilungseffect voraussichtlich von Dauer sein wird, zumal eine spätere Schrumpfung des intraskleralen Ersatzgewebes mit ihren schädlichen Folgezuständen kaum einzutreten scheint. Allerdings ist es möglich, dass beim normalen Heilungsverlauf durch das in den ersten Stunden nach der Verletzung auftretende Netzhautödem Faltenbildungen entstehen, welche sich nicht wieder ausgleichen (Fig. 5); sowie dass selbst in weiterer Entfernung vom Wundrande ohne nachweisbare 
Exp. Untersuch, über die Heilungsvorgänge von Lederhautwunden. 329

Ursache partielle Verwachsungen der Netzhaut mit der Aderhaut auftreten können; doch sind dies Seltenheiten.

Von den übrigen Wundcomplicationen, wie vor allen von den Vorfällen ist nur wenig mitzutheilen.

Die Verheilung eines Glaskörperprolapses gleicht der Organisation eines Thrombus resp. der sogenannten adhäsiven Entzïndung seröser Häute. Aus seiner zelligen Umsäumung entwickeln sich zahlreiche Zellen und Gefässspangen, um die vorgefallene Glaskörperhernie in regelloser Anordnung zu durchflechten. Hier sieht man die Karyomitosen und Gefässsprossen, sowie die Fibroblasten- und Capillarbildung am deutlichsten und übersichtlichsten während. des ganzen Heilungsprocesses. Zu bemerken ist, dass diese ungleichmässige Durchwucherung des Glaskörpers nur vor der Lederhaut, d. h. ausserhalb des Skleralniveaus zu Tage tritt.

Ein Ciliarkörperprolaps und ein Aderhautvorfall gelangten bei unseren Experimenten nicht zur histologischen Untersuchung, weil ersterer überhaupt nicht auftrat, und der letztere entweder abgetragen wurde, oder sich von selbst zurückzog.

In den seltenen Fällen von Prolaps der retinalen Wundränder spielten sich dieselben Verhältnisse im Schnittrande $\mathrm{ab}$, wie sie unter den normalen Heilungsbedingungen am inneren Wundrande sichtbar waren. Stets entwickelte sich zwischen der Retina und der Sklera ein von den anderen Häuten gebildetes Keimgewebe, um die Netzhaut von hinten her mit der Lederhaut zu verbinden. Die bindegewebige Durchwucherung der eingeklemmten Netzhautparthie erfolgte langsam aber vollständig.

Nur unter einer Bedingung wird die ausschliesslich zellig-fibrilläre Verbindung der Wundränder direct beeinträchtigt, und zwar dann, wenn Theile der äusseren Augenmuskeln interponirt sind. In solchen Fällen kann ein durch Granulationsgewebe gebildeter Wundverschluss vollkornmen unterbleiben, denn er kommt nur an den Theilen zu Stande, 
wo die Muskeln im Wundcanale fehlen, weil nur hier allein eine directe continuirliche Verbindung der Sklerafragmente durch Fibrillen geschaffen wird.

Epikrise.

Da die Uebereinstimmung unserer Befunde mit der Birch-Hirschfeld'schen Erklärung weitere Conditionalsätze entbehrlich und überflüssig erscheinen lässt, so möchte ich vor unpassend und incorrect angelegten Nähten warnen. Durch dieselben kann eine Granulationsbildung im Glaskörperraume ausserordentlich leicht zu Stande kommen, wenn wegen der vermehrten Spannung auf der äusseren skleralen Fläche die Wundränder nach innen umgestiilpt werden. Bei dem complicirten Bildungsvorgang des Keimgewebes an den Bulbushüllen gelingt es allerdings nicht immer, auf histologischem Wege die Grinde einer Granulationsbildung im Glaskörper zu ermitteln; doch ist es immerhin von grossem Werthe, durch die Erfahrungen der allgemeinen Pathologie und pathologischen Anatomie legitimirte Anhaltspunkte zu kennen, um klinisch eine solche Heilungscomplication zu vermeiden.

Bei der relativ grossen Anzahl von Versuchsthieren und dem rein experimentellen Charakter dieser Arbeit sind Hinweise auf die Heilungsvorgänge an menschlichen Augen absichtlich vermieden worden, zumal diese Untersuchungen als Grundlage für die analogen Verhältnisse menschlicher Bulbi dienen sollen, welche wir uns für eine spätere Mittheilung vorbehalten.

Es mag jetzt die Schilderung derjenigen Netzhautveränderungen folgen, welche zwar für den Heilungsvorgang der Wunden nur nebensächlich in Betracht kommen, aber für das Verständniss der weiteren im Anschluss an die Verletzung auftretenden retinalen Processe mannigfaches Interesse darbieten dürften. 
Exp. Untersuch. über die Heilungsvorgănge von Lederhautwunden. 331

\section{Mikroskopischer Befund.}

Im allgemeinen zeigt sich die Degeneration der Nervenelemente in erster Linie abhängig von der Grösse der Wunde und den unmittelbaren Folgen des operativen Eingriffes; jedoch fällt für die sorgfältige Abschätzung und Beurtheilung dieses Vorganges noch ein weiterer Factor die Lage des Schnittes - mit ins Gewicht. Je mehr nach vorn die Wunde liegt, desto rascher erfolgt die Degeneration der vorn, und desto langsamer die der hinten gelegenen nervösen Elemente. Bei äquatorialen Schnitten scheint die Degeneration nach allen Seiten hin am gleichmässigsten zu erfolgen. Bei Verwundungen in der Nähe des hinteren Poles war das Bild ausserordentlich wechselnd.

Die Degeneration der nervösen Zellen nimmt von den inneren Schichten nach den äusseren allmählich ab, und es können noch nach Monaten nahe dem Wundrande morphologisch unverändert erscheinende Zapfen und Stäbchen sichtbar sein. Wie schon erwähnt, findet man zuweilen entfernt von der Wunde ohne nachweisbare Ursache eine mehr oder weniger begrenzte Verwachsung der Aderhaut mit der Netzhaut, wodurch die letztere bogenförmig abgelöst wird. Auch kann man an solchen Stellen wiederum deutlich erkennen, dass Proliferationsproducte des Aderhautgewebes nur dann in die Retina eindringen, wenn durch die Desquamation der Pigmentepithelien und die Degeneration der äusseren Netzhautschichten die Bahn freigemacht worden ist.

Schon melurfach ist erwähnt worden, dass die Adventitia der grösseren Netzhautgefässe sich lebhaft am Wucherungsprocess betheiligen, jedoch bleiben ihnen wegen ihrer verhältnissmässig grossen gegenseitigen Abstände nur sehr kleine Bezirke übrig. Ausserdem erfährt diese Art der Zellenbildung eine weitere Einschränkung durch die eigene Verletzung. Ausser dem Collaps des peripheren Gefässendes, welches als durchschnittene Endarterie nicht mehr 
in den Dienst der Circulation gestellt werden kann, wird die Thätigkeit des centralen Blutrohres fermer dadurch sehr geschwächt, dass die im Verhältniss zum Caliberdurchschnitt auftretende retinale Blutung nicht nur eine mehr oder minder folgenschwere Gewebszerstörung zur Folge hat, sondern auch die Karyokinese zeitlich und numerisch im ungünstigen Sinne beeinflusst. Eine Ausnahme bilden auch hier wieder die Markflügel der Kaninchen, weil dieselben durch die grössere Anzahl von Gefässen ausserordentlich bevorzugt sind. Von ihnen aus entwickelt sich eine intensive und ausgiebige Gefässneubildung.

\section{Epikrise.}

Die Netzhaut hat wegen ihrer ektodermalen Anlage nur epitheliale Abkömmlinge ${ }^{1}$ ). Die Mïller'schen Faserm und die Neurogliabestandtheile unterscheiden sich von den nervösen Netzhautelementen physiologisch durch ihre minderwerthige Ausbildung, denn sie verhalten sich ,morphologisch und biologisch wie eine Bindesubstanz" ${ }^{2}$ ), indem sie in der Netzhaut den Bedingungen entsprechen, welche sie als Stützgewebe zu erfüllen haben.

Das Mesoderm wird nur durch die hineinwachsenden Gefässe repräsentirt, welche hinsichtlich der Anzahl ihrer zelligen Elemente hinter dem Stützgewebe weit zurückstehen. Andererseits besitzt ersteres unter pathologischen Verhältnissen die Fähigkeit, eine Regeneration und Production von neuem Gewebe ausgedehnter betreiben zu können. Es liegt dies in der Natur der Sache, denn die Stiitzsubstanz ist wegen ihrer epithelialen Histogenese für die Neuschaffung von Gewebe viel geringer idiodynamisch veranlagt, als das

1) W. His, Histogenese und Zusammenhang der Nervenelemente. Archiv f. Anat. a. Phys. Suppl--Bd. 1890, S. 95.

s) 0 . Weigert, Beiträge zur Kenntniss d. norm. menschlichen Neuroglia. Abhandlg. herausgeg. von d. Senckenbergischen Naturf. Gesellschaft. Bd. 19, Heft II, S. 66, 1895. 
Exp. Untersuch. über die Heilungsvorgänge von Lederhautwunden. 333

vom Mesoderm gebildete, an den meisten übrigen Körperorganen vorhandene Bindegewebe. Ganz besonders wird der Unterschied dieser beiden $Z$ wischensubstanzen dann in die Augen fallen müssen, wenn die Ernährungsbedingungen der Neuroglia ungünstig beeinflusst oder direct beschädigt sind. Nach der Anlage des Stützgewebes sind die Neurogliazellen der Netzhaut nur in der Nervenfaser- und Ganglienzellenschicht ${ }^{1}$ ) anzutreffen und daher ganz besonders schlecht geeignet, um durch isogenetische Zellproduction Continuitätstrennungen and Gewebsdefecte zur Heilung zu bringen. Damit steht im Einklang, dass auf Querschnitten die isogenetische Betheiligung der Netzhaut an der retinalen Verwachsung fast nur durch das vermehrte und verdickte Geäste der Stützfasern zum Ausdruck kommt, sowie dass regelmässig Flachschnitte nöthig werden, um das zierliche Neurogliageffecht von der compacten Zellwucherung des übrigen Keimgewebes abzugrenzen.

Der retinale Proliferationsprocess hält sich nach diesem Befunde in sehr engen Grenzen, dagegen ist, wie oben auseinandergesetzt, der Schwund an den nervösen Elementen desto ausgesprochener.

Es besteht bei vielen Autoren die Ansicht, dass die Ganglienzellen sich mitotisch vermehren können; und embryologisch ist auch Ganglienzellentheilung auf karyokinetischem Wege mit Sicherheit nachzuweisen. Desgleichen hat Edmondo Coën ${ }^{2}$ ) bei seinen Fxperimenten über die Heilung von Gehirnwunden Zelltheilungen der Ganglienzellen beschrieben. Weiter geben Baquis ${ }^{3}$ ) und Tepljaschin ${ }^{4}$ ) an, in der Ganglienzellenschicht der Retina karyokinetische

1) Greeff, Die Spinnenzellen (Neurogliazellen) im Sehnerv und in der Retina. Archiv f. Augenklinik. Bd. 29, S. 335.

2) Ed. Ooën, Ueber die Heilung ron Stichwunden des Gehims. Zieglex's Beitr. II. 1887. Ganglienzellentheilung.

3) 1. c. S. 286 .

4) 1. c, S. 389 . 
Figuren gesehen zu haben. Besonders der Letztere behauptet mit apodictischer Gewissheit, nach Retinaverletzungen ,aus der Form, der Lage und der Grösse der proliferirenden Zellen, sowie ihrer Karyomitosen" die Wucherung der Ganglienzellenschicht constatirt zu haben. Er beruft sich hauptsächlich auf das Kriterium der für die Zellen der Ganglienzellenschicht specifischen Methylenblaufärbung. Ich hielt es daher für angemessen, seine Befunde mit dieser Methode nachzuprifen, und ich habe mich derselben in der ausgiebigsten Weise unterzogen. Zugleich habe ich auch die von $\Delta$ lbrecht Bethe ${ }^{1}$ ) empfohlene intravasculäre $\mathrm{Me}$ thylenblauinjection, sowie die Nissl'sche ${ }^{2}$ ) Methode benutzt, um dieser Frage näher zu treten, resp. sie zur Lösung zu bringen. Niemals ist es mir gegliickt, etwas derartiges zu finden. Wohl liegen Verwechselungen und Täuschungen ausserordentlich nahe, weil in den benachbarten Räumen der Ganglienzellen sich häufig Kerne finden, doch möchte ich mich für eine Proliferation der Ganglienzellen nur dann aussprechen, wenn neben den karyokinetischen Figuren die charakteristischen morphologischen Eigenschaften ihres Protoplasmas sichtbar sind. Da mir ein solcher Befund an den in Theilung begriffenen Zellen trotz der mannigfachen Methoden und der zahlreichen Experimente nicht geglïckt ist, so bin ich gezwungen, die Mittheilungen dieser Autoren als eine Art histologischer Inconsequenz aufzufassen, zumal gerade in den inneren Schichten des der Wunde benachbarten Netzhautdistriktes nervöse Elemente stets vermisst werden (Fig. 3, 5, 8). Von den Autoren wird als Grund für die Annahme der Zelltheilung das fast ausschliessliche Vorkommen ron Monasteren angeführt. Sie betrachteten diese Kerntheilung als Reactionserscheinung, besonders da in einem solchen Monaster häufig

1) Archiv f. mikr. Anatomie. 1895. Bd. 44, S. 579.

2) Tageblatt der 58. Versammlung deutscher Naturforscher Aerzte. Strassburg 1885. S. 506. 
Exp. Untersuch. über die Heilungsvorgänge von Lederhautwunden. 335

Fetttropfen zu finden waren, welche als Zeichen des Degenerationsbeginns gedeutet wurden. Nach diesen Angaben müsste man schliessen, dass eine Kerntheilung stattfände, welche vor der vollständigen Zelltheilung mit dem Untergange der Zellen abschlösse. Da ich selbst nie etwas derartiges zu beobachten Gelegenheit hatte, so muss ich mich auch diesen Mittheilungen gegenüber reservirt verhalten. Andererseits will ich diese Möglichkeit der unvollständigen Zelltheilung nicht direct in Abrede stellen. Insofern stimmen unsere Ergebnisse mit den Befunden bei Gehirnerkrankungen utberein, als Coën und besonders v. Kahlden ${ }^{1}$ ) das hier neugebildete Gewebe zum grössten Theil aus den Gefässwandungen ableiten.

\section{Erklärung der Abbildungen auf Tafel III-V.}

Fig. 1. Kaninchen. 10 Stunden. Vorderer Augenabschnitt. Netzhaut nicht gezeichnet.

Links ist die Chorioidea prolabirt; rechts ist die Aderhaut und das episklerale Gewebe am Wundrande operativ entfernt. Stadium der Entzündung und der Coagulationsnekrose. Ep.=episkleralen Gewebe. $S=$ Sklera. $C h=$ Chorioidea. $G=$ Glaskörper. $a=$ Randstellung der Leukocyten. $b=$ Blutungen. $c$ und $d=$ eindringende Leukocyten in die skleralen Saftlücken.

Fig. 2. Kaninchen. Vorderer Augenabschnitt. 50 Stunden.

Ueberall Beginn der Proliferation. Degeneration in Suprachorioidea und Retina. $E p .=S$. wie vorher. $C h 1=$ Suprachorioidea. $C h 2=$ Gefässschichten. $\quad C h 3=$ Pigmentepithelzellen. $\quad R=$ Retina. $R 1=$ Zapfen und Stäbchen. $R 2=$ Körnerschichten. $R 3=$ Ganglienzellenschicht. $a=$ Blutungen. $b=$ Leukocyten, zum Theil mit Pigment. $\quad c=$ Granulationszellen ohne, $d=$ mit Pigment. $e=$ Proliferationszellen der Aderhaut. $f=$ Karyomitosen der Suprachorioidea, $g=$ desquam. Pigrnentepithelzellen. $h=$ äussere Körner. $i=$ Reste vom Pigmentepithel. $k=$ degenerirte Zapfen und Stäbchen. $l=$ Proliferationszellen in der Retina. $m=$ Stützfasern. $n=$ degenerirende Ganglienzellen. $o=$ desquam. Endothelien d. Suprachorioidea. $p=$ freies Pigment. $q=$ Karyomitosen.

Fig. 3. Kaninchen. 4. Tag. Vorderer Augenabschnitt.

1) v. Kahlden, Ueber die Heilung von Gehirnwunden. Centralblatt. f. allgem. Pathol. Bd. 2. Nr. 18, S. 751. 
336 E. Krückmann, Unters. üb. Heilungsvorgängev. Lederhautwunden.

Fast gleichmässige Betheiligung des äusseren und inneren Keimgewebes. Degeneration der Retina. $M$ : Muskel, $E p ., S, C h, R$ wie früher. $a=$ Proliferationszellen der Nervenfaserschicht im Markflügel. $b=$ gewucherte Adrentitiazellen. $c=$ Neurogliazellen. $d=$ varicöse Nervenfasern. $e=$ desquam. Pigmentepithelzellen. $f=$ leere Lücken von $g=$ degenerirten Ganglienzellen. $h=$ Neubildung ron Gefässen. $i=$ pigmenthaltige Proliferationszellen. $k=$ junge Capillaren. $l=$ Eindringen der Keimzellen in die Sklera. $m=$ episklerale Keimzellen.

Fig. 4. Katze. Vord. Augenabschnitt. Netzhaut nicht gezeichnet.

20. Tag. Fntwicklung des Keimgewebes. Degeneration der Zellen des tapetum lucidum. Ep., $S, C h$ wie vorher. $a=$ episklerales, $b=$ chorioideales Keimgewebe. $c=$ degenerirende, $d=$ normale Tapetumzellen.

Fig. 5. Kaninchen, weiss. Hinterer Augenabschnitt mit Verletzung der Markflügel. 5. Tag.

Ueberwiegen des inneren Keimgewebes. Proliferation und Degeneration in der Retina. $E p, M, S, O h, R$ wie vorher, $a=$ verdickte Stützfasern. $b=$ Lücken von $c=$ degenerirten Ganglienzellen. $d=$ Karyomitose in einer Stützfaser. $e=$ Proliferationszellen in der Sklera $f=$ in der Retina. $g=$ retinale Capillaren mit gewacherten Adventitiazellen. $h=$ Neurogliazellen,$i=$ neugebildete Zellen $u . k=$ Capillaren der Retina. $l=$ ef. $b$ : in den Markflügeln. $m=$ sklerale Spindelzellen.

Fig. 6. Ratte. 15 Tage. Hinterer Augenabschnitt. Netzhaut nicht gezeichnet.

Ueberwiegen des inneren Keimgewebes mit Betheiligung der Suprachorioidea. $E_{p}, S, C h$ wie rorher. $a=$ Spindelzellen der Tenon'schen Kapsel. $b=$ eindringende Keimzellen in die Sklera. $c=$ Proliferationszellen in der Tenon'schen Kapsel. $d, f=$ pigmenthaltige Proliferationszellen. $e=$ präformirte Skleralzellen. $g=$ neugebildete Zellen der Suprachorioidea.

Hund. $\left\{\begin{array}{l}\text { Fig. 7. 21 Tage. Narbe u. Muskelschwand im Ciliarkörper. } \\ \text { Fig. 8. } 60 \text { Tage. Narbe der Netzhaut und der Aderhant. }\end{array}\right.$

Umformung des Frsatzgewebes in Spindelzellen und Fibrillen. Betheiligung der Suprachoriojdea.

Fig. 7. $E p, S$ wie vorher. $S u p .=$ Suprachorioidea. $C i t .=$ Ciliarkörper. Ers. = Ersatzgewebe. $a=$ normale, $d=$ verbreiterte intermuskuläre Septen. $b=$ normale, $c=$ atrophische Muskelbündel, $e=\mathrm{Be}$ theiligung der Suprachorioidea, $f=$ pigmenthaltige Zellen. $g=$ collabirte Capillaren.

Fig. 8. Ep., $S$, Sup., $C h, R$, Ers. wie vorher. $a=$ verdickte Stützfasern. $b=$ Neuroglia. $c=$ Betheiligung der Suprachorioidea. $d=\mathrm{Li}-$ mitansendothelien. $d^{\prime}=$ vielleicht Limitansendothelien. 


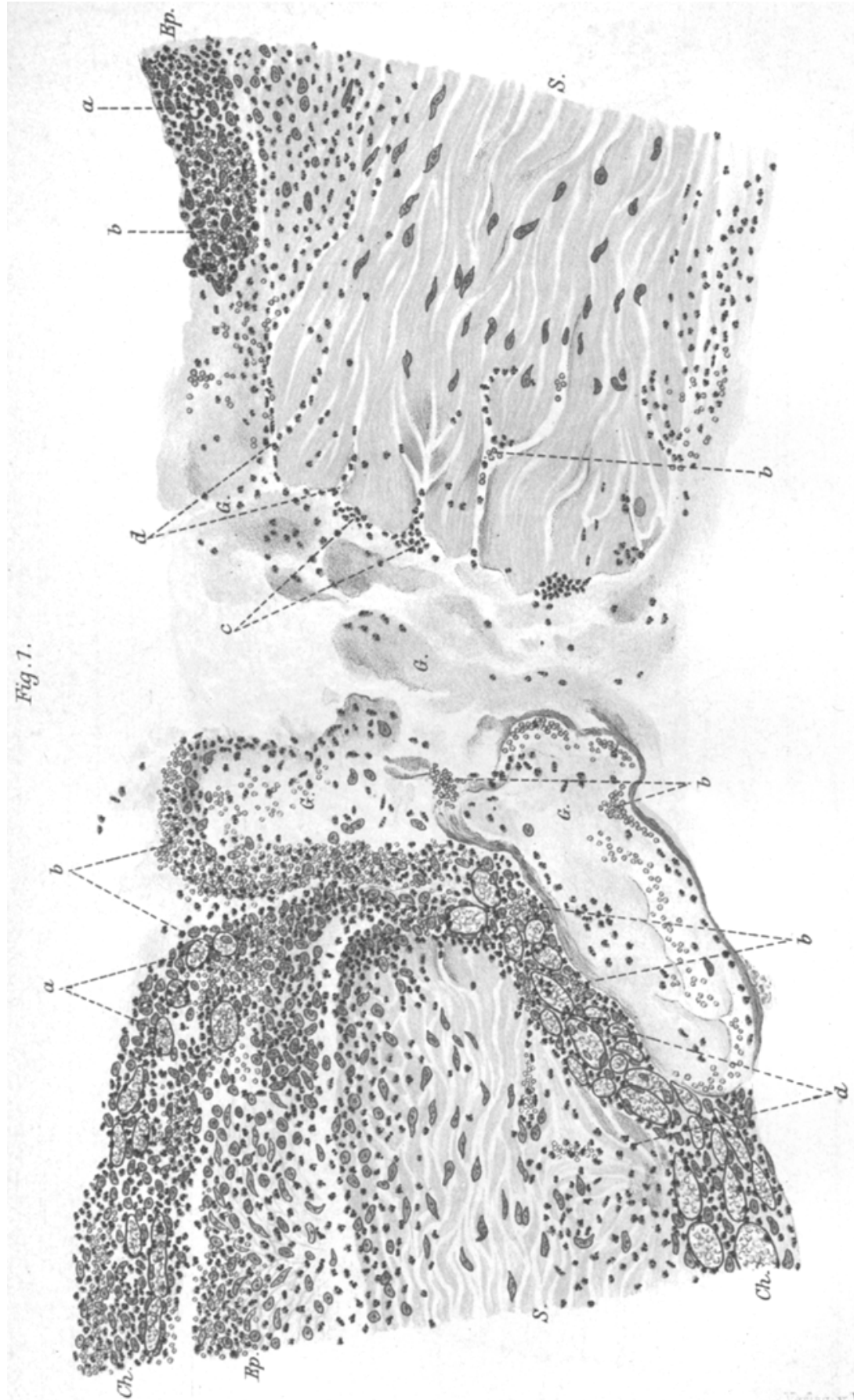



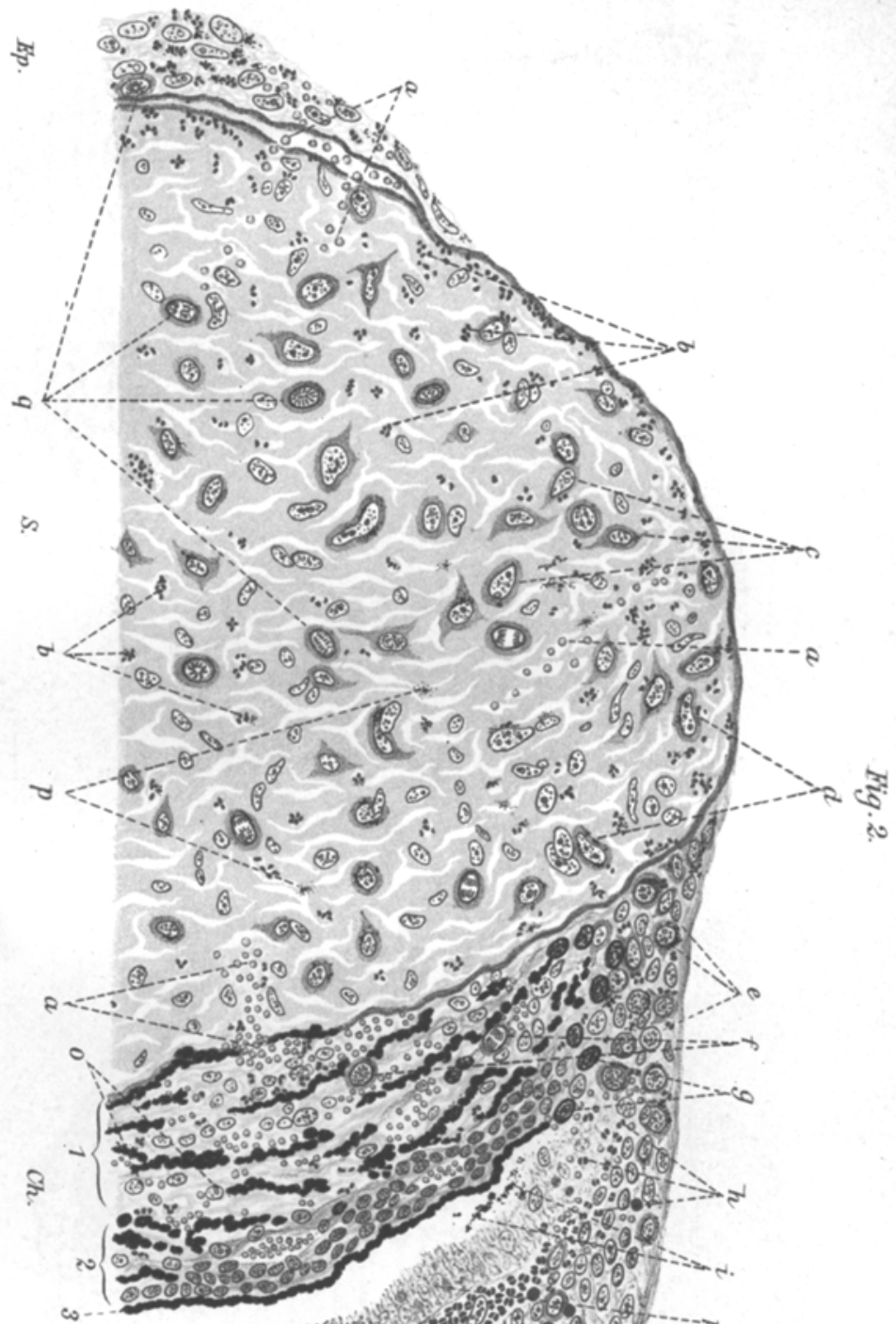

a

- ygh whing

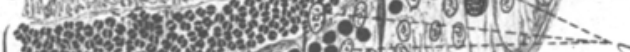

$\{$ 象

co
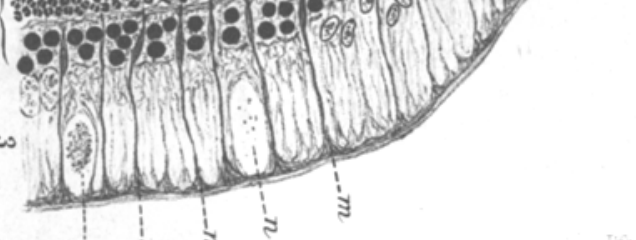

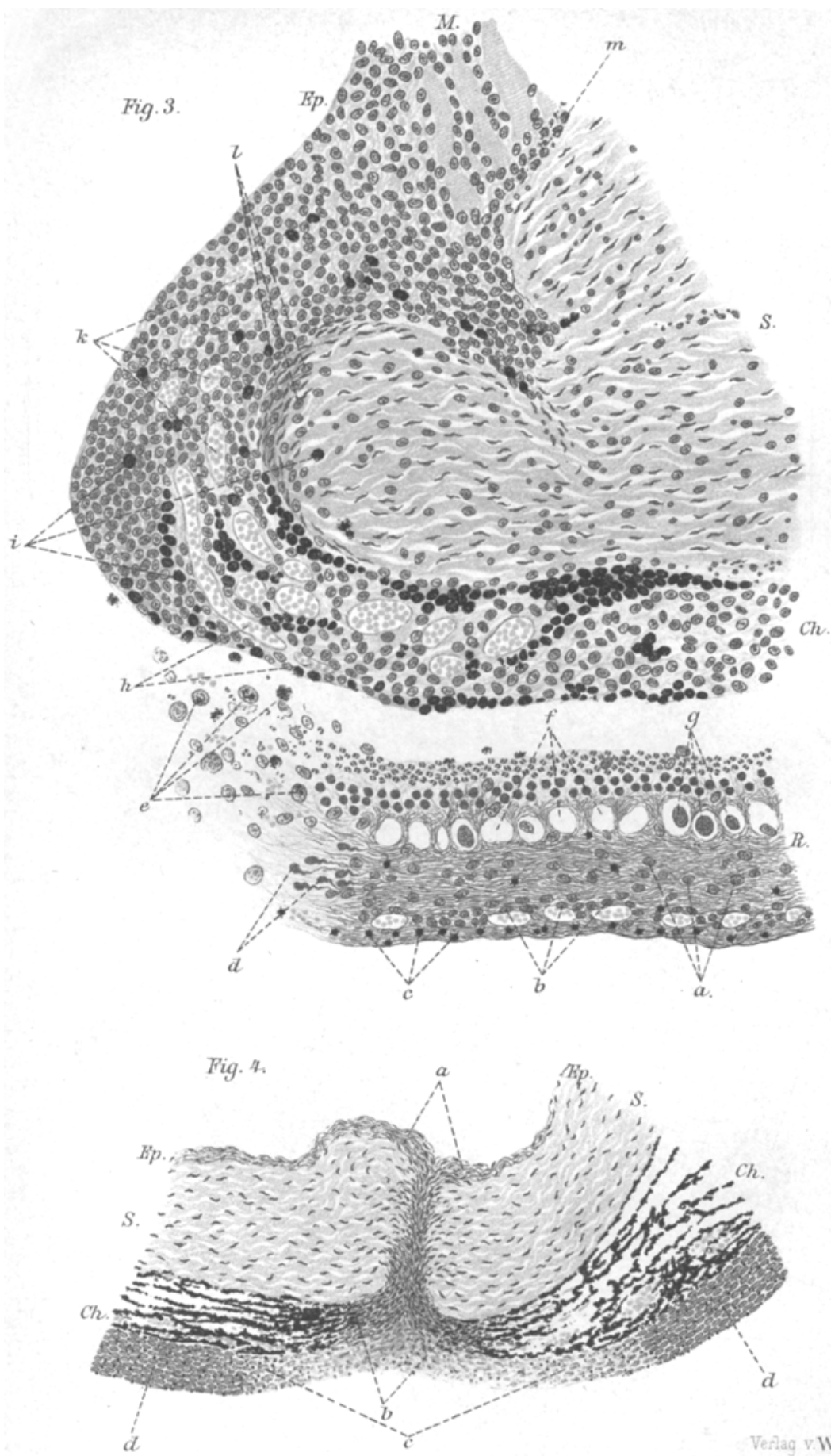


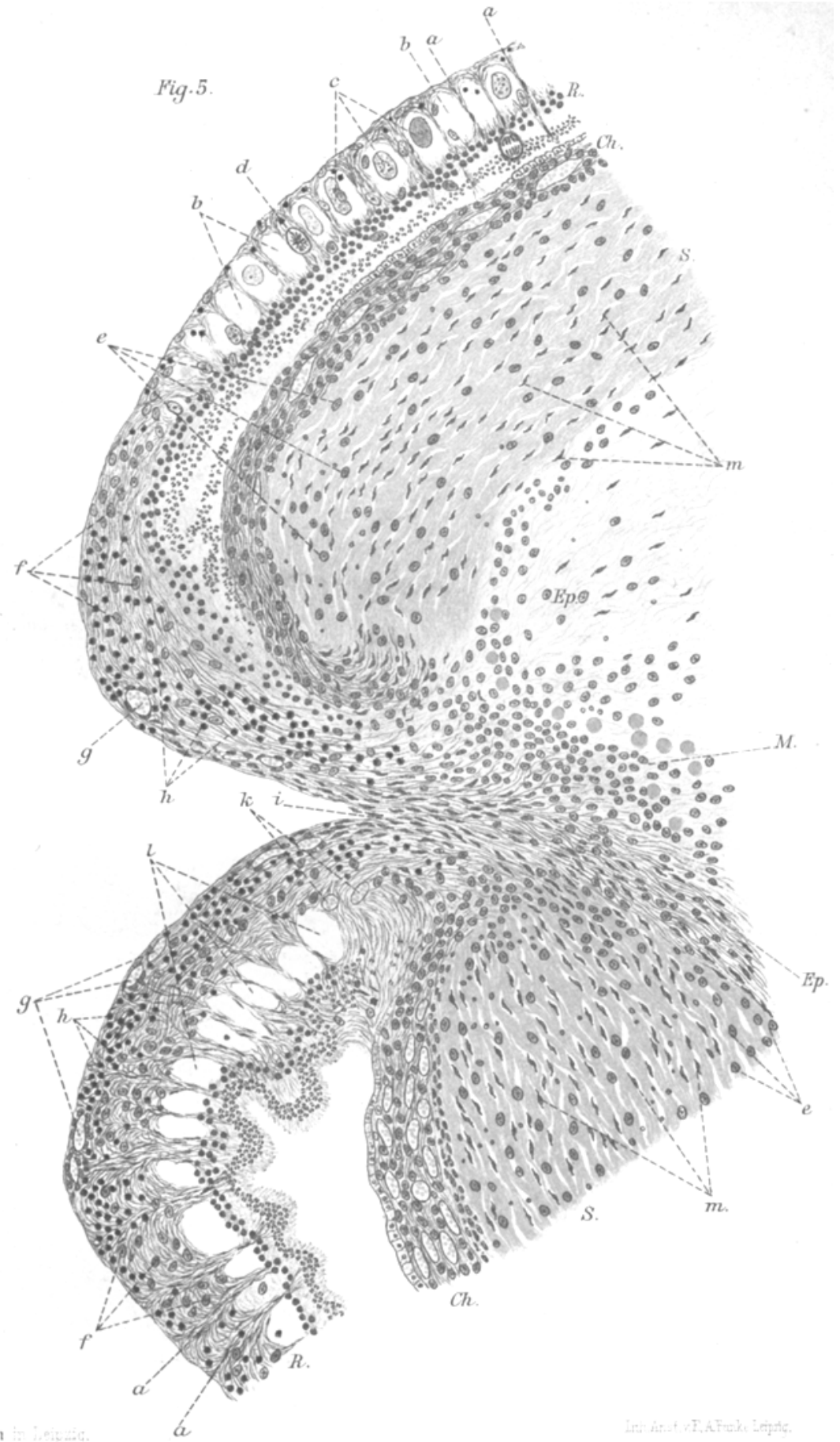




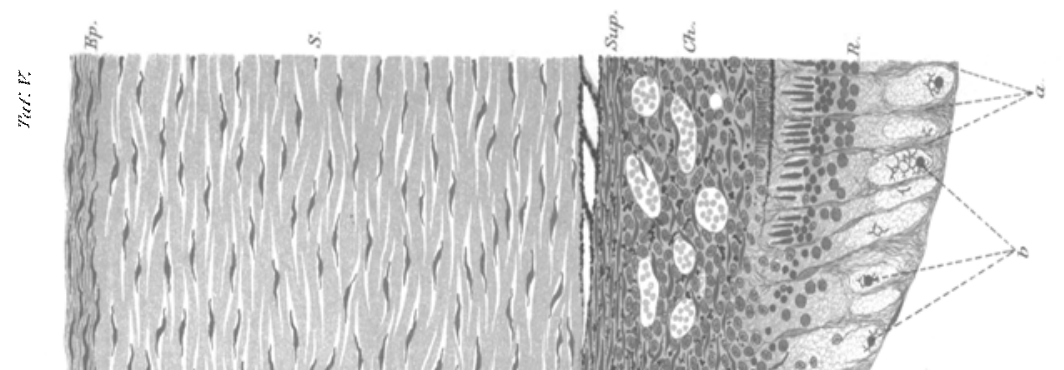

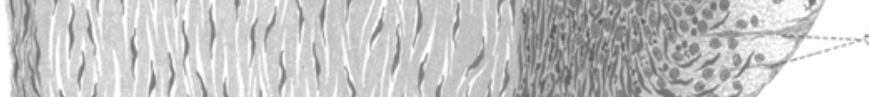

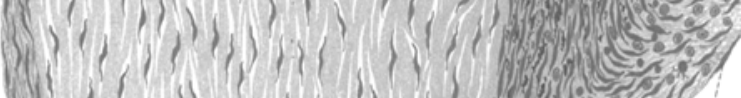
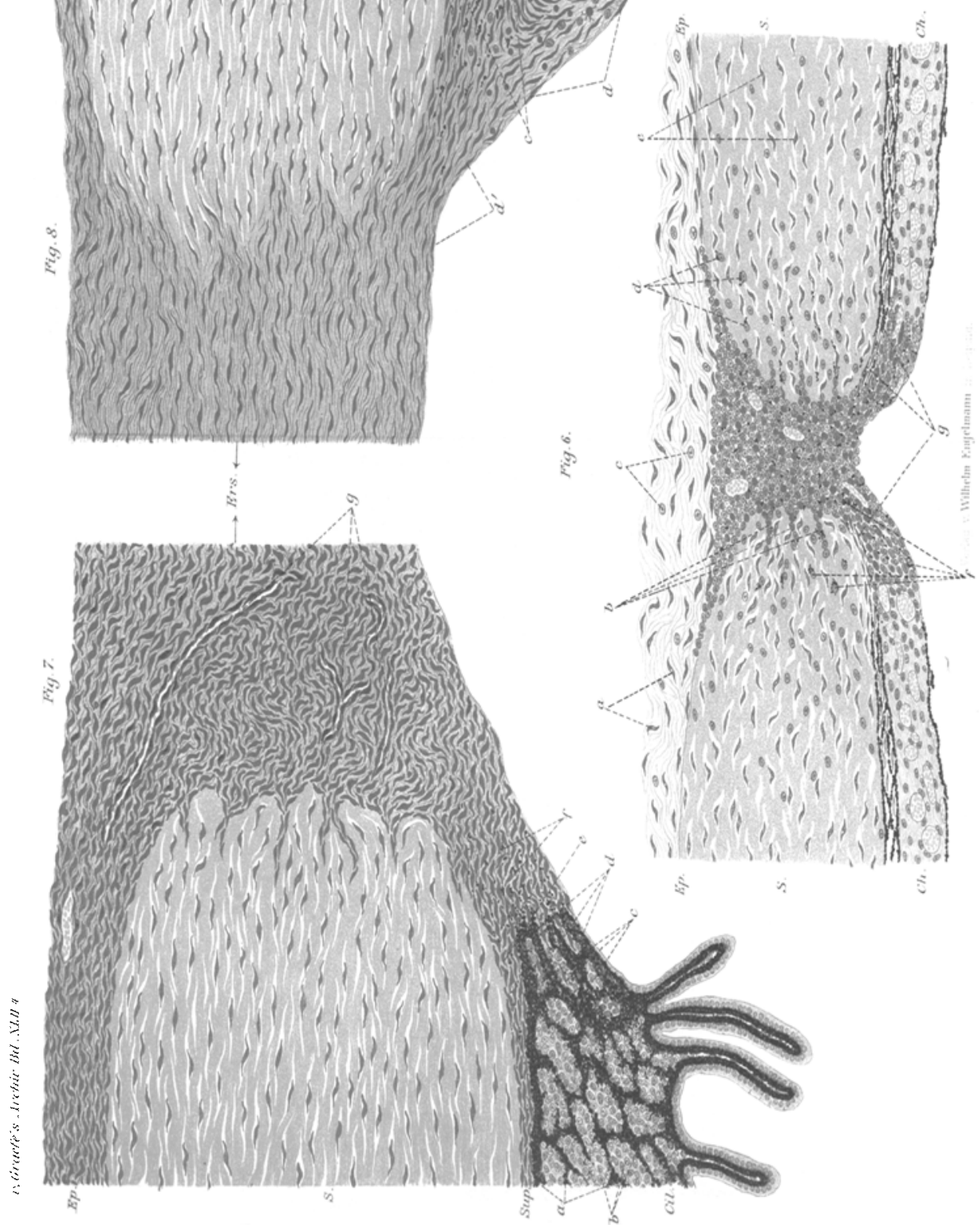